\title{
THE CHEMICAL COMPOSITION OF CARBON-RICH, VERY METAL POOR STARS: A NEW CLASS OF MILDLY CARBON RICH OBJECTS WITHOUT EXCESS OF NEUTRON-CAPTURE ELEMENTS
}

\author{
Wako Aoki, ${ }^{1}$ John E. Norris, ${ }^{2}$ SeAn G. Ryan, ${ }^{3}$ Timothy C. BeErs, ${ }^{4}$ AND Hiroyasu ANDo ${ }^{1}$ \\ Received 2001 September 14; accepted 2001 November 16
}

\begin{abstract}
We report on an analysis of the chemical composition of five carbon-rich, very metal poor stars based on high-resolution spectra. One star, CS 22948-027, exhibits very large overabundances of carbon, nitrogen, and the neutron-capture elements, as found in the previous study of Hill et al. This result can be interpreted as a consequence of mass transfer from a binary companion that previously evolved through the asymptotic giant branch stage. By way of contrast, the other four stars we investigate exhibit no overabundances of barium $([\mathrm{Ba} / \mathrm{Fe}]<0)$, while three of them have mildly enhanced carbon and/or nitrogen $([\mathrm{C}+\mathrm{N}] \sim+1)$. We have been unable to determine accurate carbon and nitrogen abundances for the remaining star (CS 30312-100). These stars are rather similar to the carbon-rich, neutron-captureelement-poor star CS 22957-027 discussed previously by Norris et al., although the carbon overabundance in this object is significantly larger $([\mathrm{C} / \mathrm{Fe}]=+2.2)$. Our results imply that these carbon-rich objects with "normal" neutron-capture element abundances are not rare among very metal-deficient stars. One possible process to explain this phenomenon is as a result of helium-shell flashes near the base of the asymptotic giant branch in very low metallicity, low-mass $\left(M \lesssim 1 M_{\odot}\right)$ stars, as recently proposed by Fujimoto et al. The moderate carbon enhancements reported here $([\mathrm{C} / \mathrm{Fe}] \sim+1)$ are similar to those reported in the famous $r$-process-enhanced star CS 22892-052. We discuss the possibility that the same process might be responsible for this similarity, as well as the implication that a completely independent phenomenon was responsible for the large $r$-process enhancement in CS 22892-052.
\end{abstract}

Subject headings: nuclear reactions, nucleosynthesis, abundances - stars: abundances stars: AGB and post-AGB - stars: carbon - stars: Population II

On-line material: machine-readable table

\section{INTRODUCTION}

Many observational and theoretical studies have been undertaken to clarify the formation and chemical evolution of the Galaxy, as well as the nature of stellar evolution at the earliest times. For this purpose, extensive surveys of metal-deficient stars have been carried out (e.g., the HK survey of Beers, Preston, \& Shectman 1992; Beers 1999), which have identified objects as metal poor as $[\mathrm{Fe} /$ $\mathrm{H}]=-4 .{ }^{5}$ One unexpected result of the HK survey is that many $(\sim 10 \%-15 \%)$ of the most metal poor stars exhibit anomalously strong $\mathrm{CH}$ bands, most easily understood in terms of an excess of carbon in their atmospheres. Moreover, the trend of carbon overabundances appears to increase with declining metallicity (Rossi, Beers, \& Sneden 1999), that is, the most metal-deficient stars exhibit the highest level of carbon overabundances.

Recently, high-resolution abundance studies have been extended to include these carbon-rich metal-poor stars, but the number of such studies is still quite limited. Given that stars with carbon excesses are numerous among the most metal deficient stars, they take on considerable importance

\footnotetext{
${ }^{1}$ National Astronomical Observatory, Mitaka, Tokyo 181-8588, Japan; aoki.wako@nao.ac.jp, ando@optik.mtk.nao.ac.jp.

${ }^{2}$ Research School of Astronomy and Astrophysics, The Australian National University, Private Bag, Weston Creek Post Office, Canberra, ACT 2611, Australia; jen@mso.anu.edu.au.

${ }^{3}$ Department of Physics and Astronomy, The Open University, Walton Hall, Milton Keynes, MK7 6AA, UK; s.g.ryan@open.ac.uk.

${ }^{4}$ Department of Physics and Astronomy, Michigan State University, East Lansing, MI 48824-1116; beers@pa.msu.edu.

${ }^{5}[A / B] \equiv \log \left(N_{A} / N_{B}\right)-\log \left(N_{A} / N_{B}\right)_{\odot}, \quad$ and $\log \epsilon_{A} \equiv \log \left(N_{A} / N_{\mathrm{H}}\right)$ +12 for elements $A$ and $B$.
}

in the study of the chemical evolution of the Galaxy. For instance, a recent study of chemical enrichment in the early Galaxy (Abia et al. 2001) suggests that the initial mass function at zero metallicity must peak at intermediate stellar mass in order to account for the carbon and nitrogen enhancement in extremely metal poor stars.

One explanation for the enhancement of carbon in some of the very metal poor stars is that put forward to account for the moderately metal poor classical $\mathrm{CH}$ stars $([\mathrm{Fe} /$ $\mathrm{H}] \sim-1.5$ ). These are usually explained by a model involving mass transfer from a carbon-enhanced asymptotic giant branch (AGB) star (one that has since evolved to the white dwarf stage and cannot now be seen) to its lower mass companion, which is now observed as a $\mathrm{CH}$ star (McClure \& Woodsworth 1990). In this case, a carbon-rich metaldeficient star should exhibit the chemical composition of AGB stars (i.e., evolved intermediate-mass stars) at the earliest times. One distinct feature of these stars is the enhancement of the $s$-process elements. The most well studied example of the phenomenon is LP 625-44, for which the abundances of 16 heavy elements, including three elements at the $s$-process peaks $(\mathrm{Sr}, \mathrm{Ba}$, and $\mathrm{Pb})$, have been determined (Aoki et al. 2000). The variation of radial velocity shown by that work confirms the binarity of this object, and strongly supports the above mass-transfer scenario. However, this analysis revealed a quite different abundance trend from previous theoretical expectations, in particular concerning the $\mathrm{Pb} / \mathrm{Ba}$ abundance ratio. In metal-poor $\mathrm{AGB}$ stars, the $s$-process was expected to build up lead, due to the paucity of seed nuclei compared to the (primary) neutrons, giving a high $\mathrm{Pb} / \mathrm{Ba}$ ratio (e.g., Gallino et al. 1998). However, this picture is modified on a star-by-star basis by variations in the concentration of ${ }^{13} \mathrm{C}$ from which the neu- 
trons originate (Busso, Galino, \& Wasserburg 1999), giving rise to a range of possible $\mathrm{Pb} / \mathrm{Ba}$ values (Ryan et al. 2001). Aoki et al. (2001) showed that the abundance pattern of LP 625-44 is well reproduced by a parametric model of the $s$-process with neutron exposure $\tau \sim 0.7$. Modeling of AGB nucleosynthesis and evolution where such physical conditions are produced will promote our understanding of the $s$-process and AGB evolution at low metallicity.

On the other hand, one carbon-rich, very metal poor star, CS 22892-052, exhibits an extreme excess of $r$-process, instead of s-process, elements (Sneden et al. 1996, 2000). The relationship, if any, between carbon production and $r$ process enhancement is unclear, but the mechanism of carbon enrichment may well be quite different from the scenario of mass transfer from an s-process-rich AGB star. The only other currently recognized extremely $r$-process enhanced metal-poor star, CS 31082-001 (Cayrel et al. 2001), exhibits no excess of carbon, and hence demonstrates that the two phenomena are not required to be linked to one another. Furthermore, Norris, Ryan, \& Beers (1997b; see also Bonifacio et al. 1998) showed that the carbonenhanced, very metal poor $([\mathrm{Fe} / \mathrm{H}]=-3.4)$ star CS 22957027 exhibits no enhancement of neutron-capture elements. These facts indicate that the canonical mass-transfer scenario cannot generally explain all carbon-rich objects.

Recently, another mechanism for carbon enrichment was suggested by Preston \& Sneden (2001) to account for their observations of a number of metal-poor subgiants that do not exhibit clear signs of binarity. These authors reported on a long-term radial velocity program for carbonenhanced metal-poor stars, and found that none of the three carbon-rich, metal-poor subgiants in their sample exhibited velocity variations exceeding $0.5 \mathrm{~km} \mathrm{~s}^{-1}$ over an 8 year period. They suggested that these stars might have undergone an enhanced mixing event at the end of their giantbranch evolution that "recycled" them to the base of the subgiant branch, because of increased hydrogen mixing into their cores, a scenario originally suggested by Bond (1974) to explain the subgiant $\mathrm{CH}$ stars he discovered. It should also be noted that the subgiants studied by Preston \& Sneden (2001) have enhanced s-process elements.

Elemental abundance studies of metal-poor carbon-rich stars without excesses of $s$-process elements are quite limited, and the origin of their carbon enhancement is still unclear. One recent hypothesis for the formation of these stars is that of Fujimoto, Ikeda, \& Iben (2000), who suggested that production of large carbon and nitrogen excesses may follow from extensive mixing in low-mass
$\left(M \lesssim 1 M_{\odot}\right)$, very metal poor stars at their helium core flash at the end of the giant branch, or during the helium-shell flash phase on the lower AGB.

Thus, the carbon-rich, very metal poor stars show a wide variety of elemental abundance patterns, and several alternative explanations of their carbon enrichment must be considered, in particular for objects without excesses of neutron-capture elements. In the present paper we report an abundance analysis based on high-resolution spectroscopy for five carbon-rich, very metal poor stars. In $\S 2$ we summarize the observations, report measurements of equivalent widths for detectable spectral lines, and discuss the determination of radial velocities. Estimation of the atmospheric parameters for our program stars is described in $\S 3$, where the effect of carbon and nitrogen excesses on the determination of effective temperatures is also discussed. The analysis and results are provided in $\S 4$. In $\S 5$ we summarize the characteristic chemical compositions of our program stars and discuss possible interpretations of these results.

\section{OBSERVATIONS AND MEASUREMENTS}

We selected our program sample from the lists of metalpoor stars verified by medium-resolution spectroscopic follow-up of candidates from the HK survey (Beers et al. 1992; Beers 1999), taking into account their brightness, metallicity, and apparent level of carbon enhancement. We used the GP index, as defined in Table 2 of Beers et al. (1992), as an indicator of carbon enhancement. The GP index is a pseudo equivalent width of the $\mathrm{CH}$ molecular band around $4300 \AA$ ( $\mathrm{G}$ band) measured in the mediumresolution spectra. Our program stars were taken to be those objects with GP indices that were at least twice the typical values of other metal-poor stars from the HK survey with similar metallicity and colors. Our sample is listed in Table 1, along with additional information concerning the observations. We note that the GP indices of CS 30314-067 and CS 29502-092 were newly measured from more recently obtained medium-resolution spectra.

\subsection{Observations and Data Reduction}

Observations were made with the University College London coudé échelle spectrograph (UCLES) at the AngloAustralian $3.9 \mathrm{~m}$ Telescope during the period 1994-1998. The observing log is given in Table 1 . For most observations, the Tektronix $1024 \times 1024 \mathrm{CCD}$ was used as the detector; the MIT-LL $2048 \times 4096$ CCD was used during the 1998 April run. The spectra were taken with a resolving power $R \approx 40,000$. Column (1) of Table 1 lists the star

TABLE 1

Program Stars and ObServations

\begin{tabular}{|c|c|c|c|c|c|c|c|c|c|}
\hline $\begin{array}{l}\text { Star } \\
(1)\end{array}$ & $\begin{array}{c}V \\
(2)\end{array}$ & $\begin{array}{l}B-V \\
(3)\end{array}$ & $\begin{array}{l}\text { GP } \\
(\AA) \\
(4)\end{array}$ & $\begin{array}{c}\text { Wavelength } \\
\text { (Å) } \\
(5)\end{array}$ & $\begin{array}{c}\text { Exposure }^{a} \\
\text { (minutes) } \\
(6)\end{array}$ & $\begin{array}{l}\mathrm{S} / \mathrm{N} \\
(7)\end{array}$ & $\begin{array}{c}\sigma_{W} \\
(\mathrm{~m} \AA) \\
(8)\end{array}$ & $\begin{array}{c}V_{\mathrm{rad}} \\
\left(\mathrm{km} \mathrm{s}^{-1}\right) \\
(9)\end{array}$ & $\begin{array}{c}\text { Observation Date (JD) } \\
\text { (10) }\end{array}$ \\
\hline CS $22948-027 \ldots \ldots$ & 12.66 & 1.13 & 7.79 & $5010-8530$ & $75(3)$ & 51 & 6.7 & $-63.33 \pm 0.56$ & 1994 Jun (2449520) \\
\hline \multirow[t]{2}{*}{ CS $30314-067 \ldots \ldots$} & 11.85 & 1.13 & 5.80 & $3740-4800$ & $120(4)$ & 77 & 3.9 & & 1997 Aug \\
\hline & & & & $4970-8400$ & $60(2)$ & 95 & 4.0 & $+145.58 \pm 0.29$ & 1997 Aug (2450684) \\
\hline \multirow[t]{2}{*}{ CS $22877-001 \ldots \ldots$} & 12.20 & 0.73 & 4.54 & $3620-4880$ & $120(4)$ & 36 & 5.6 & & 1998 Apr \\
\hline & & & & $4400-7710$ & $60(2)$ & 77 & 4.6 & $+166.12 \pm 0.25$ & 1998 Apr (2450914) \\
\hline CS $30312-100 \ldots \ldots$ & 12.95 & 0.59 & 3.49 & $4440-7710$ & $120(4)$ & 57 & 5.1 & $-128.91 \pm 0.28$ & 1998 Apr (2450914) \\
\hline \multirow[t]{2}{*}{ CS $29502-092 \ldots \ldots$} & 11.88 & 0.77 & 4.51 & $3740-4800$ & $150(5)$ & 70 & 4.1 & & 1998 Aug \\
\hline & & & & $4600-7130$ & $60(2)$ & 73 & 4.0 & $-67.35 \pm 0.27$ & 1998 Aug (2451039) \\
\hline
\end{tabular}

a Number of exposures is given in the parentheses. 
name. Columns (2) and (3) list the $V$ apparent magnitude and $B-V$ color reported in previous HK survey papers. The GP index for each star is listed in column (4).

Data reduction was accomplished using standard procedures within the IRAF ${ }^{6}$ suite, including bias subtraction, flat-fielding, background subtraction, and wavelength calibration. Cosmic-ray removal was done by comparing two or more frames obtained during different exposures for each star, using the same spectrograph setup. The wavelength intervals, exposure times, and numbers of exposures (in parentheses) for each of our program stars are listed in columns (5) and (6) of Table 1.

Examples of the spectra in the wavelength region around $4550 \AA$ are shown in Figure 1, along with identifications of the prominent absorption lines. The absorption lines of the heavy elements, especially the Ba II $\lambda 4554$ feature, are discussed in the following sections. For comparison, the spectrum of an s-process-rich, metal-poor star, LP 625-44, on which we reported separately (Aoki et al. 2000, 2001), is also shown in the figure.

In order to assess the quality of the data, we constructed a summed spectrum and a difference spectrum from two spectra obtained by different exposures for each object. The signal-to-noise ratio $(\mathrm{S} / \mathrm{N})$ achieved was based on the signal level of the summed spectrum for the nearly line free region near the peak of échelle blaze profile, compared with the standard deviation of the difference spectrum for the same region. Column (7) of Table 1 lists the $\mathrm{S} / \mathrm{N}$ at $4350 \AA$ for the blue setup, and those at $6000 \AA$ for the red one. These values are comparable to the square root of the number of photons obtained at each of the listed wavelengths.

\subsection{Measurement of Equivalent Widths}

Equivalent widths of the extracted and wavelengthcalibrated spectra were measured by fitting Gaussian profiles to the absorption lines. The local continuum levels were estimated interactively during the fitting procedure. In order to reject lines that may suffer from contamination by other features, we checked the atomic line list provided by Kurucz \& Bell (1995), and the line list of $\mathrm{CH}$ and CN molecules used in the present work (see $\S 4.2$ ). The equivalent

${ }^{6}$ IRAF is distributed by the National Optical Astronomy Observatories, which is operated by the Association of Universities for Research in Astronomy, Inc. under cooperative agreement with the National Science Foundation. widths measured for individual atomic lines are listed in Table 2.

Random (internal) errors in the equivalent widths were estimated by comparing two or more measurements of those lines in the spectra obtained with individual exposures. The standard errors for lines with equivalent widths smaller than $60 \mathrm{~m} \AA$ are provided in column (8) of Table 1 $\left(\sigma_{W}\right)$. These internal errors will be used below for estimating abundance uncertainties, and for estimating upper limits on abundances for elements with no detectable spectral lines.

We checked the dependence of $\sigma_{W}$ on line strength for CS 30314-067 and CS 29502-092, in which a number of strong and weak lines were measured. The discrepancy between the $\sigma_{W}$ derived from the weak lines $(W<60 \mathrm{~m} \AA)$ and that derived from stronger lines is neither significant nor systematic: $\Delta \sigma_{W}$ (strong - weak) $=-0.9$ and $+0.3 \mathrm{~m} \AA$ for $C S$ 30314-067 and CS 29502-092, respectively. We note that the uncertainty in the derived elemental abundance (at fixed $\sigma_{W}$ ) for strong lines is generally larger than that obtained for weak lines, due to the saturation effect on line absorption.

The estimated $\sigma_{W}$ is dependent on the $\mathrm{S} / \mathrm{N}$ of the spectrum at each given wavelength; the $\mathrm{S} / \mathrm{N}$ at the edge of the échelle blaze function is lower than at the center. However, the free spectral range is well covered for the blue region, in which most of the lines used in the present analysis are found, and the $\mathrm{S} / \mathrm{N}$ is not severely dependent on the line position in the échelle order after combining the spectra for neighboring orders. On the other hand, the $\mathrm{S} / \mathrm{N}$ of the spectra in the bluest regions is considerably lower than at intermediate wavelengths. We have calculated $\sigma_{W}$ for lines with $\lambda<4000 \AA$ for CS 30314-067 and CS 29502-092, in which many iron lines with $\lambda<4000 \AA$ were detected. The $\sigma_{W}$ derived is roughly $2 \mathrm{~m} \AA$ higher than that obtained from lines in the longer wavelength regions. This indicates that we underestimate the uncertainty in the resulted abundances (by a maximum of 50\%) for some elements (e.g., $\mathrm{Mg}$, $\mathrm{Ti}$, and $\mathrm{Fe}$ ) for which the analysis includes lines with $\lambda<4000 \AA$. However, the error arising from $\sigma_{W}$ is not the dominant factor in the total uncertainty in the resulting abundances for these elements (see $\S 4.4$ ); hence, we simply adopted the $\sigma_{W}$ given in Table 1 for the purpose of error estimation.

\subsection{Measurement of Radial Velocities}

Some of the carbon-rich, very metal poor stars may be accounted for by mass transfer across a binary system, as

TABLE 2

EQUivalent WidThS FOR Program Stars (mÅ)

\begin{tabular}{ccccccccc}
\hline \hline $\begin{array}{c}\lambda \\
(\AA)\end{array}$ & $\begin{array}{c}\chi \\
(\mathrm{eV})\end{array}$ & $\log g f$ & $30314-067$ & $29502-092$ & $22877-001$ & $30312-100$ & $22948-027$ & $g f$ Reference \\
\hline Na I: & & & & & & & & \\
5889.95 & 0.000 & 0.11 & 165 & 135 & 118 & 158 & 209 & 1 \\
5895.92 & 0.000 & -0.19 & 153 & 97 & 95 & 130 & 195 & 1 \\
Mg I: & & & & & & & & \\
3829.35 & 2.710 & -0.48 & 197 & $\ldots$ & 133 & $\ldots$ & $\ldots$ & $2-0.25$ \\
3832.30 & 2.710 & -0.13 & 215 & 169 & 173 & $\ldots$ & $\ldots$ & $2-0.25$ \\
3838.30 & 2.720 & -0.10 & 203 & 181 & 218 & $\ldots$ & $\ldots$ & $3-1.05$ \\
4571.10 & 0.000 & -5.61 & 89 & 21 & 21 & $\ldots$ & $\ldots$ & 4 \\
\hline
\end{tabular}

NotE.-Table 2 is published in its entirety in the electronic edition of the Astrophysical Journal. A portion is shown here for guidance regarding its form and content.

REFERENCES.-(1) Wiese \& Martin 1980; (2) Peterson \& Carney 1989; (3) Luck \& Bond 1981; (4) Thévenin 1989. 

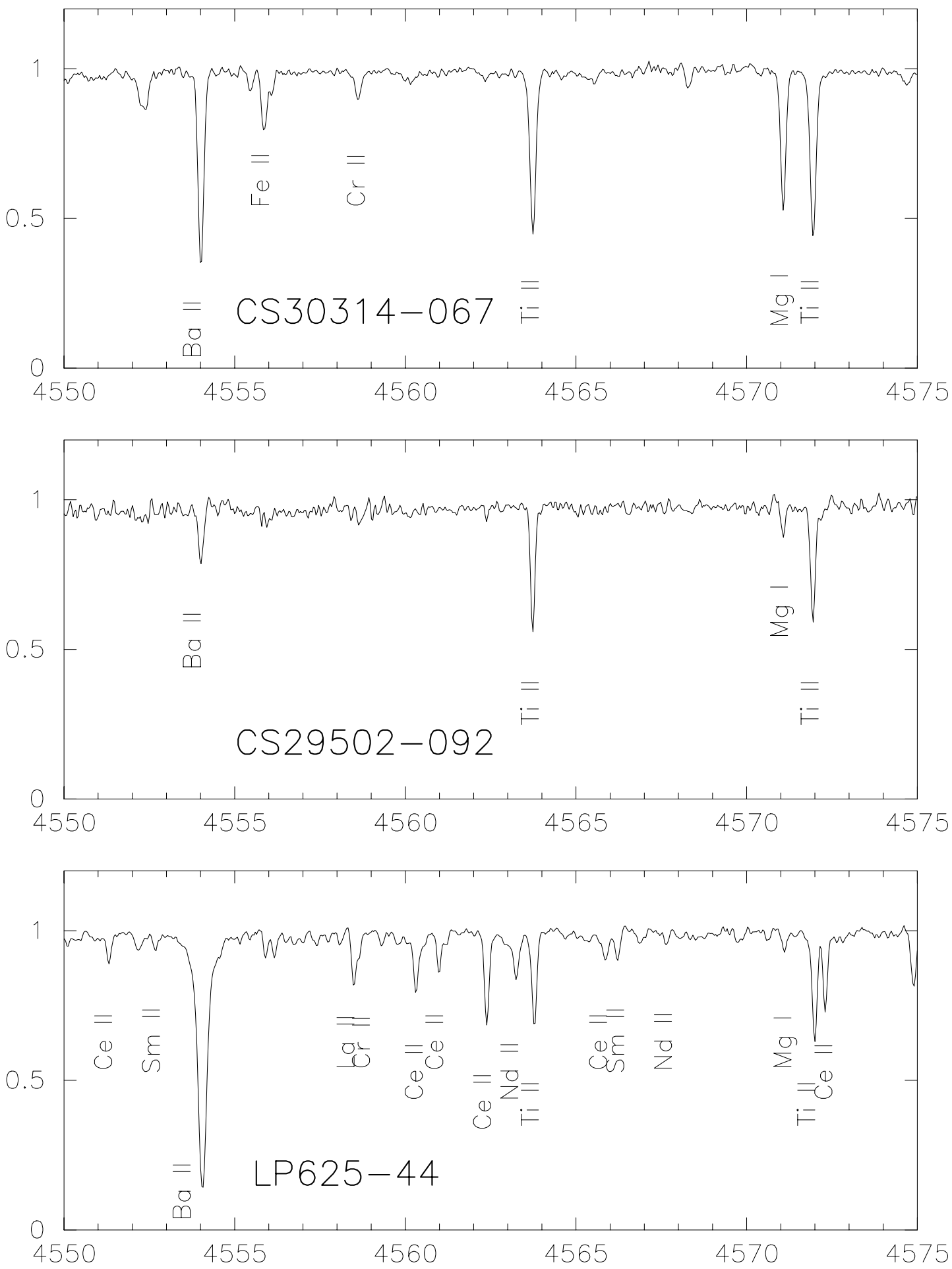

Wavelength $(A)$

FIG. 1.-Observed spectra near the Ba II $\lambda 4554$ line. The spectra of two giants (CS 30314-067 and CS 29502-092) are compared with that of the $s$-process-rich subgiant LP 625-44 (Aoki et al. 2000). The Ba II 24554 lines in the two giants are much weaker than that in LP 625-44. No lines of other neutron-capture elements are detected in the two giants, while numerous lines of La II, Ce II, Nd II, and Sm II appear in LP 625-44.

mentioned in $\S 1$. Hence, a search for radial velocity variation is quite important for a test of this scenario. Since we have observed each object at only one epoch, we cannot discuss the variation of radial velocities here, but these measurements provide a baseline against which future studies can be compared.
For the measurement of radial velocity, we selected clean $\mathrm{Fe}$ I lines between 4800 and $5400 \AA$ for which the data quality is good and blending with $\mathrm{CH}$ and $\mathrm{CN}$ molecular lines is not severe. The line position was measured by fitting Gaussian profiles for about 20 lines, then deriving the heliocentric radial velocity. Results are provided in column (9) of 
Table 1, along with the standard error in each measurement. For reference, we also list the Julian date (JD) of the observations in column (10) of Table 1.

Radial velocity variation has already been established for CS 22948-027 by Preston \& Sneden (2001), who also provided orbital parameters (e.g., $P=505$ days) for this object. Our result $\left(V_{\mathrm{rad}}=-63.3 \mathrm{~km} \mathrm{~s}^{-1}\right)$ is in good agreement with the value expected on JD $=2449520$ from their orbital parameters, and confirms the binarity of this star.

\section{STELLAR PARAMETERS}

In order to perform a detailed abundance analysis using a model-atmosphere approach, we must first determine the appropriate atmospheric parameters, i.e., the effective temperature, surface gravity, metallicity, and microturbulence, to be applied. Strong molecular absorption due to carbon (and nitrogen) can affect the determination of effective temperatures obtained from comparison with broadband photometry. Below we report a method for determination of effective temperature that takes these effects into consideration in some detail ( $\$ 3.1)$. The determination of other atmospheric parameters is discussed in $\S 3.2$.

\subsection{Effective Temperatures for Carbon-Enhanced Stars}

Accurate estimation of effective temperature is crucial for accurate measurement of elemental abundances. Effective temperatures are frequently determined from the comparison of broadband colors (e.g., $B-V$ ) with temperature scales predicted by model atmospheres (e.g., Bessell, Castelli, \& Plez 1998). However, for carbon-rich objects the temperature scale of normal (oxygen-rich) stars cannot necessarily be applied, because of the strong absorption bands of carbon-bearing molecules, such as $\mathrm{CH}, \mathrm{CN}$, and $\mathrm{C}_{2}$, which can severely affect the emergent flux distribution. Strong $\mathrm{CH}$ bands, for instance, which are the defining characteristics of carbon-rich, metal-poor stars such as those in our program, reduce the flux in the $B$ band, and hence increase (redden) the $B-V$ color compared to normal metal-poor stars of the same effective temperature. In addition to carbon, nitrogen is sometimes found to be overabundant in carbon-rich stars, and can also affect the emergent flux. For these reasons, it is necessary to consider an effective temperature scale that includes the effects of carbon and nitrogen excess of varying amounts.

To evaluate the effect of molecular absorption on the spectra of metal-poor stars, we calculated synthetic spectra that included molecular lines, as well as strong atomic lines, in the wavelength region 3000-10000 $\AA$. About 20,000 strong $\mathrm{Mg}, \mathrm{Ca}, \mathrm{Ti}$, and $\mathrm{Fe}$ lines were selected from the line list of Kurucz \& Bell (1995). Balmer lines of hydrogen were also included in the calculation, as were molecular lines due to $\mathrm{MgH}$ and $\mathrm{SiH}$ listed in Kurucz (1993b). The lines arising from the CH $A^{2} \Delta-X^{2} \Pi$ and $B^{2} \Sigma^{-}-X^{2} \Pi$ bands, the CN violet and red systems, and the $\mathrm{C}_{2}$ Swan system, explained in $\S 4.2$, were used in addition. The total number of molecular lines is about 55,000 .

As a check on the suitability of the synthetic spectra obtained by this approach, we calculated the spectra for several metallicities with solar abundance ratios (i.e., $[\mathrm{C} /$ $\mathrm{Fe}]=[\mathrm{N} / \mathrm{Fe}]=0$ ), using the model atmospheres of Kurucz (1993a), and compared them with Kurucz's emergent fluxes, calculated taking into account huge numbers of atomic and molecular lines. Although the number of lines in our list is obviously insufficient to reproduce Kurucz's emergent fluxes for solar metallicity $([\mathrm{M} / \mathrm{H}]=0)$, the fluxes for $[M / H] \leq-2$ are well reproduced by our synthetic spectra. We conclude that our line list is adequate to calculate the colors of the metal-poor stars discussed in the present work.

Synthetic spectra were calculated for several levels of carbon and nitrogen enhancement, using the above line list, for models with metallicity $[\mathrm{M} / \mathrm{H}]=-2$ and -3 , and for $T_{\text {eff }}$ ranging from 4000 to $6000 \mathrm{~K}$. Calculations were done for $T_{\text {eff }}=4000,4500,5000$, and $5500 \mathrm{~K}$ for $\log g=2.0$; $T_{\text {eff }}=5000,5500$, and $6000 \mathrm{~K}$ for $\log g=3.0$; and $T_{\text {eff }}=$ 5500 and $6000 \mathrm{~K}$ for $\log g=4.0$. The model atmospheres by Kurucz (1993a) were used for this purpose. Then, fluxes for several photometric bands (Johnson-Cousins system; Bessell 1990) were obtained from the synthetic spectra. The calculated $B-V$ and $R-I$ values are shown as a function of model effective temperature in Figure 2. We show the result for $\log g=3.0$ for $T_{\text {eff }}=6000 \mathrm{~K}$, and those for $\log g=2.0$ for $T_{\text {eff }}=4000,4500,5000$, and $5500 \mathrm{~K}$ in this figure.

The effect of surface gravity is not large in these parameter ranges. The difference in $B-V$ for $\log g=3.0$ and for $\log g=4.0$ is $0.024 \mathrm{mag}$ for $[\mathrm{Fe} / \mathrm{H}]=-3.0,[\mathrm{C} / \mathrm{H}]=[\mathrm{N} /$ $\mathrm{H}]=-1.0$, and $T_{\text {eff }}=6000 \mathrm{~K}$. For other cases, the impact of changes in surface gravity on $B-V$ is even less; the effect on $R-I$ is negligible.

As can be seen in Figure 2, $B-V$ is larger (redder), for higher $[\mathrm{C} / \mathrm{H}]$ and $[\mathrm{N} / \mathrm{H}]$, at a given effective temperature. This is because the emergent flux in the $B$ band is severely affected by $\mathrm{CH}$ and $\mathrm{CN}$ bands, while the $\mathrm{C}_{2}$ absorption in the $V$ band is not strong, except for cool stars $\left(T_{\text {eff }} \lesssim 4500\right.$ $\mathrm{K})$ with extremely high $[\mathrm{C} / \mathrm{Fe}]$. On the other hand, $R-I$ is smaller (bluer) for higher $[\mathrm{C} / \mathrm{H}]$ and $[\mathrm{N} / \mathrm{H}]$ at a given effective temperature. The $\mathrm{CN}$ red system is important for the red and near-infrared regions in carbon-rich stars, and the effect should be larger in the $I$ band than in the $R$ band. As can be seen in this figure, the effect of carbon and nitrogen excess is much smaller in $R-I$ for $T_{\text {eff }} \leq 5000 \mathrm{~K}$; in such cases the $R-I$ color is preferable for the determination of effective temperature. In the present work, however, only the $B-V$ colors were available for most of the program stars; hence, we make use of the effective temperature scale based on those values. Future measurements of $R-I$ colors would be most useful.

In the above calculations, model atmospheres produced for solar elemental abundance ratios $([\mathrm{X} / \mathrm{Fe}]=0)$ were used. However, the effect of carbon and nitrogen excesses on atmospheric structure should also be considered in the model calculation, because the absorption due to many molecular lines blocks the flux, and the atmosphere heats up because of the back-warming effect. In this sense our calculation is incomplete, in particular for cool objects. We expect that this effect will have only a small impact, however, for stars with effective temperatures in the range considered in the present work.

The model atmospheres and effective temperature scale of metal-poor stars with carbon and nitrogen excess were also studied by Hill et al. (2000). The $B-V$ and $R-I$ colors provided by Hill et al. (2000), as a function of effective temperature, are also shown in Figure 2 for the same set of chemical compositions assumed, i.e., $[\mathrm{Fe} / \mathrm{H}]=-3$ and $[\mathrm{C} /$ $\mathrm{H}]=[\mathrm{N} / \mathrm{H}]=-1$. Since the effect of carbon and nitrogen excess on the atmospheric structures is taken into account in their calculation, their result may be more realistic than ours. Nevertheless, our colors, derived for $[\mathrm{Fe} / \mathrm{H}]=-3$ 


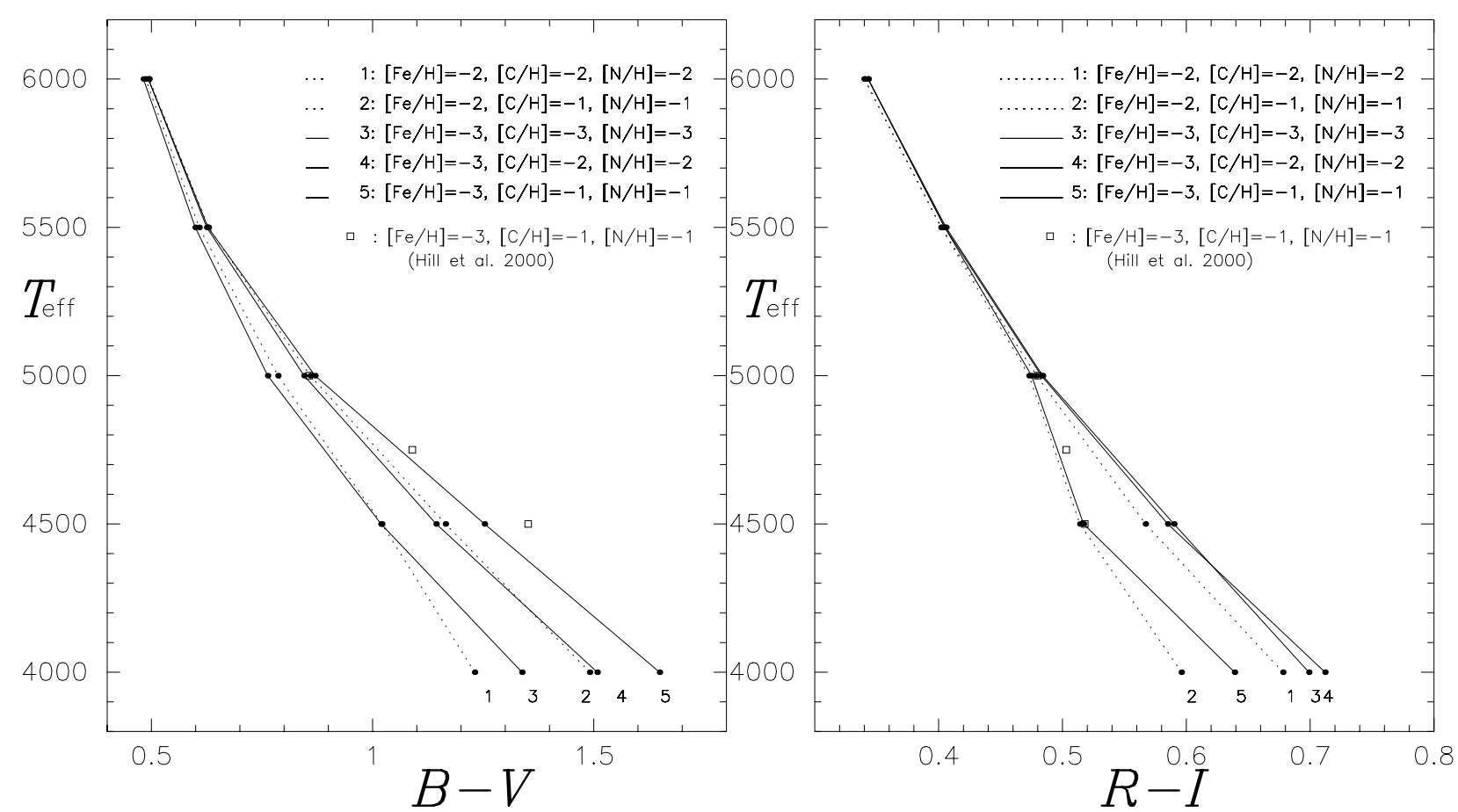

Fig. 2.-Predicted colors $(B-V$ and $R-I)$ calculated using synthetic spectra (see text). The colors calculated by Hill et al. (2000) are plotted for comparison.

and $T_{\text {eff }} \gtrsim 4500 \mathrm{~K}$, agree well with those obtained by Hill et al. (2000; see Fig. 2). This supports the supposition above that the effect of carbon and nitrogen enhancement on the atmospheric structure is not large for stars with effective temperature higher than $4500 \mathrm{~K}$. For stars with lower temperatures our scale may be less than optimal. We note, however, that the above procedure significantly improves the estimation of the effective temperatures of carbon-rich stars, and should be taken into account, especially when $B-V$ is the only available color.

Typical errors in the reported photometry results in an uncertainty $\Delta(B-V)$ of about 0.02 mag (Beers, Preston, \& Shectman 1985). Several of our program stars may suffer from interstellar reddening of up to $0.05 \mathrm{mag}$. The $\Delta T_{\text {eff }} /$ $\Delta(B-V)$, in the temperature region of interest, is $160-200$ $\mathrm{K} / 0.1 \mathrm{mag}$. Hence, a typical uncertainty in the $T_{\text {eff }}$ estimation is about $100 \mathrm{~K}$. The effect of this uncertainty on elemental abundance determinations is estimated in $\S 4.4$ below.

The effect of carbon and nitrogen excess on the determination of effective temperatures is quite important for our two coolest stars, CS 30314-067 and CS 22948-027. At $B-V=1.13$, the reddest color for stars in our sample, the effective temperature derived for the assumption of $[\mathrm{Fe} /$ $\mathrm{H}]=-3$ and $[\mathrm{C} / \mathrm{H}]=[\mathrm{N} / \mathrm{H}]=-1$ is higher by about $400 \mathrm{~K}$ than that obtained when no excess of carbon or nitrogen is present. We iterated the determination of effective temperature and the abundance analysis of carbon and nitrogen for each object until consistency between the resulting abundance and the assumed one in the analysis was achieved.

CS 22948-027 was also studied by Preston \& Sneden (2001), who derived an effective temperature including the blanketing effects by $\mathrm{CH}$ and $\mathrm{CN}$ bands. Their effective temperature is $4640 \mathrm{~K}$, which agrees very well with our estimation (4600 K). Like us, they did not include the backwarming effect on the model atmosphere. The effective temperature of about $4600 \mathrm{~K}$ derived by the present study and Preston \& Sneden (2001) is lower than the result of Hill et al. (2000), $T_{\text {eff }}=4800 \mathrm{~K}$, who included the back-warming effect in the model calculation. This discrepancy may be due to the effect of carbon enhancement on atmospheric structure.

After the present analysis was completed, we became aware of new results for near-infrared photometry listed in the Second Incremental Release Point Source Catalog (PSC) from the 2MASS survey for three of our stars. ${ }^{7}$ These new data enable us to estimate the effective temperature from other colors, such as $V-K$, which are less sensitive to line blanketing than $B-V$. We applied the temperature scale for $V-K$ produced by Alonso, Arribas, \& MartínezRoger (1999), using the 2MASS $K$ photometry $(K=9.03 \pm 0.03,10.03 \pm 0.03$, and $9.60 \pm 0.03$ for CS 30314-067, CS 22877-001, and CS 29502-092, respectively, where the magnitudes and errors are the "default" values provided in the PSC). The derived effective temperatures are 4320,4920 , and $4800 \mathrm{~K}$, respectively. These temperatures are systematically lower than those derived from $B-V$ in the present analysis, although the disagreement is not severe $(80-200 \mathrm{~K})$. We do not pursue this issue further here, but investigation of this discrepancy, e.g., from studies of blanketing in the near-infrared region, will be valuable for more reliable estimation of effective temperatures for carbon-rich objects in the future.

\footnotetext{
${ }^{7}$ This research has made use of the NASA/IPAC Infrared Science Archive, which is operated by the Jet Propulsion Laboratory, California Institute of Technology, under contract to the National Aeronautics and Space Administration.
} 
TABLE 3

Stellar Parameters

\begin{tabular}{|c|c|c|c|c|c|c|}
\hline Star & $\begin{array}{l}T_{\text {eff }} \\
(\mathrm{K})\end{array}$ & $\log g$ & $\begin{array}{c}v_{\text {tur }} \\
\left(\mathrm{km} \mathrm{s}^{-1}\right)\end{array}$ & {$[\mathrm{Fe} / \mathrm{H}]$} & {$[\mathrm{C} / \mathrm{H}]$} & {$[\mathrm{N} / \mathrm{H}]$} \\
\hline CS $22948-027 \ldots \ldots$ & 4600 & 1.0 & 2.2 & -2.57 & -0.6 & -0.8 \\
\hline CS $30314-067 \ldots \ldots$ & 4400 & 0.7 & 2.5 & -2.85 & -2.4 & -1.7 \\
\hline CS $22877-001 \ldots \ldots$ & 5100 & 2.2 & 2.0 & -2.72 & -1.7 & -2.7 \\
\hline CS $30312-100 \ldots \ldots$ & 5500 & 3.0 & 2.3 & -2.17 & $\ldots$ & $\ldots$ \\
\hline CS $29502-092 \ldots . .$. & 5000 & 2.1 & 1.8 & -2.76 & -1.8 & -2.1 \\
\hline
\end{tabular}

\subsection{Surface Gravity, Metallicity, and Microturbulence}

We began our analysis assuming $\log g=2.0$ for all of our stars. Then the surface gravities were revised to obtain ionization balance between $\mathrm{Fe} \mathrm{I}$ and $\mathrm{Fe}$ II lines. We assumed LTE in the parameter estimates as well as in the abundance analysis. The non-LTE effect is important in warm, lowgravity stars, but is expected to be negligible in subgiants and cool giants studied here (e.g., Gratton et al. 1999). The final derived surface gravities are listed in Table 3. Note that CS 30312-100 has the gravity of a subgiant, while the other four stars appear to be giants. Metal abundances were initially assumed to be $[\mathrm{M} / \mathrm{H}]=-2.5$, then revised to the $[\mathrm{Fe} / \mathrm{H}]$ values derived from the abundance analysis for $\mathrm{Fe}$ I and $\mathrm{Fe}$ II lines. These parameters were also revised once the effective temperature was redetermined, taking the excess of carbon and nitrogen into account, as described in $\$ 3.1$.

The microturbulent velocities $\left(v_{\text {tur }}\right)$ were determined from the Fe I lines by demanding no dependence of derived abundance on equivalent widths. For CS 22948-027, we also measured the microturbulent velocity using the $\mathrm{CN}$ lines with measurable equivalent widths, and confirmed the agreement with that determined from the $\mathrm{Fe}$ I lines. The effect of microturbulent velocity on the atmospheric structure was neglected, and the ATLAS9 model atmospheres with $v_{\text {tur }}=2 \mathrm{~km} \mathrm{~s}^{-1}$ were used for the abundance analysis, with the microturbulence reset to the values listed in Table 3.

\section{ABUNDANCE ANALYSIS AND RESULTS}

The elemental abundance analysis was performed using the model atmospheres in the ATLAS9 grid of Kurucz (1993a). The standard analysis, based on the equivalent widths measured in $\S 2.2$, was obtained for most species, employing the LTE spectral synthesis code used in Aoki \& Tsuji (1997a). As in Ryan, Norris, \& Beers (1996), Unsöld's (1955) treatment of van der Waal's broadening, enhanced by a factor of 2.2 in $\gamma$, was used. To check our calculations of elemental abundances, we analyzed some metallic species in the metal-poor $([\mathrm{Fe} / \mathrm{H}] \sim-2.7)$ giant $\mathrm{HD} 122563$ (Norris, Ryan, \& Beers 1996) using Kurucz's LTE code WIDTH6, as well as with our own software. The results agree well; differences in derived abundances are generally smaller than 0.02 dex for weak lines $(W<80 \mathrm{~m} \AA)$. There is a systematic discrepancy of derived abundances based on strong lines between the two analyses ( $\lesssim 0.10 \mathrm{dex})$, which is likely due to the differences of the treatment of the line broadening (see Ryan 1998). Since most elemental abundances in the present work relied exclusively on weak lines, the influence on the final results is negligible. Exceptions are the derived abundances of $\mathrm{Na}, \mathrm{Al}, \mathrm{Si}$, and $\mathrm{Sr}$, which relied on strong lines.

In addition to the above standard analysis, the spectrum synthesis method was applied to some metallic lines that are affected by blending. The abundances of carbon and nitrogen were also determined by spectrum synthesis of the $\mathrm{CH}$, $\mathrm{C}_{2}$, and $\mathrm{CN}$ bands. Carbon isotope ratios were derived from analysis of the $\mathrm{CH}$ and $\mathrm{CN}$ lines.

The analysis was started for $\mathrm{Fe} \mathrm{I}$ and $\mathrm{Fe}$ II lines using the assumed stellar parameters discussed above. From the analysis of iron lines, the microturbulence was determined, and surface gravity and metallicity were revised. The carbon and nitrogen abundances were derived using these (revised) stellar parameters. After the abundances of $\mathrm{C}, \mathrm{N}$, and $\mathrm{Fe}$ were tentatively determined by this procedure, the effective temperature was revised making use of the effective temperature scale described in $\S 3$. This procedure was iterated until consistency between the derived $\mathrm{C}$ and $\mathrm{N}$ abundances, and those assumed for the analysis, was achieved.

\subsection{Atomic Lines}

The standard analysis, based on equivalent widths, was made for most atomic lines without contamination with other lines. The atomic line data compiled by Norris et al. (1996) was used for most elements. We supplemented this list with additional lines, especially for the wavelength region $3700-3760 \AA$, as well as for the red spectral regions. In the star CS 22948-027, many lines of neutron-capture elements have been detected. The line list of neutroncapture elements produced from the same sources referred to by Aoki et al. (2001) was applied to the analysis of this star. The references for the $g f$-values are given in Table 2.

The effect of hyperfine splitting on the lines of Mn (4030, 4033, and $4034 \AA)$ and Co $(3845,3873,3894$, and $4121 \AA)$ was taken into consideration using the estimation procedure shown in Figure 1 of Ryan et al. (1996).

The results of our abundance analysis are listed in Table 4. One important conclusion is that the abundances of heavy neutron-capture elements $(Z \geq 38)$ are quite low for four of our stars, in contrast to CS 22948-027, which exhibits a large excess of these elements. Figure 1 shows portions of spectra around $\mathrm{Ba}$ II $\lambda 4554$ for two of the Ba-poor stars (CS 30314-067 and CS 29502-092) and the $s$-process-rich star LP 625-44 (Aoki et al. 2000). Note that the $\mathrm{Ti}$ II and $\mathrm{Fe}$ II lines for the two (giant) Ba-poor stars are stronger than those in (the subgiant) LP 625-44, due to their lower temperatures and gravities. By way of contrast, the Ba II $\lambda 4554$ lines in the two giants are much weaker than that seen in LP 625-44. No lines of other neutron-capture elements are detected in the two giants, while numerous lines of La II, Ce II, Nd II, and Sm II appear in LP 625-44. Figure 1 clearly shows the low abundances of neutroncapture elements in the stars studied in the present work. This result is discussed in detail in $\S 5$.

Our spectrum of CS 22948-027 includes Eu II $\lambda 6645$, and a $\mathrm{Eu}$ abundance $[\mathrm{Eu} / \mathrm{Fe}]=+1.57$ was derived from this line. However, this result is rather uncertain because of possible contamination from molecular absorption lines. No clear $\mathrm{Eu}$ line was detected in the spectra of our other program stars. Nevertheless, upper limits on Eu abundance are still useful, because the $\mathrm{Ba} / \mathrm{Eu}$ ratio may allow us to distinguish the origin of heavy elements in these stars, i.e., whether they are to be primarily associated with the $r$ process or with the s-process. We estimated upper limits on Eu abundance for CS 30314-067, CS 29502-092, and CS 22877-001 from the Eu II $\lambda 4129$, taking $3 \sigma_{W}$ at this wave- 
TABLE 4

[Fe/H] and Relative Abundance, [X/Fe]

\begin{tabular}{|c|c|c|c|c|c|c|c|c|c|c|c|c|c|c|c|}
\hline \multirow[b]{2}{*}{ ELEMENT } & \multicolumn{3}{|c|}{ CS 30314-067 } & \multicolumn{3}{|c|}{ CS 29502-092 } & \multicolumn{3}{|c|}{ CS 22877-001 } & \multicolumn{3}{|c|}{ CS $30312-100$} & \multicolumn{3}{|c|}{ CS 22948-027 } \\
\hline & {$[\mathrm{X} / \mathrm{Fe}]$} & $n$ & SE & {$[\mathrm{X} / \mathrm{Fe}]$} & $n$ & SE & {$[\mathrm{X} / \mathrm{Fe}]$} & $n$ & SE & {$[\mathrm{X} / \mathrm{Fe}]$} & $n$ & SE & {$[\mathrm{X} / \mathrm{Fe}]$} & $n$ & SE \\
\hline $\mathrm{C} \ldots \ldots \ldots \ldots$ & +0.5 & & 0.18 & +1.0 & & 0.18 & +1.0 & & 0.18 & $\ldots$ & & & +2.0 & & 0.18 \\
\hline $\mathrm{N} \ldots \ldots \ldots \ldots$ & +1.2 & & 0.24 & +0.7 & & 0.24 & 0.0 & & 0.24 & $\ldots$ & & & +1.8 & & 0.24 \\
\hline $\mathrm{C}+\mathrm{N} \ldots \ldots$ & +0.8 & & & +1.0 & & & +0.9 & & & $\ldots$ & & & +2.0 & & \\
\hline $\mathrm{Na} \mathrm{I}_{1} \ldots \ldots \ldots$ & -0.08 & 2 & 0.18 & -0.01 & 2 & 0.18 & -0.24 & 2 & 0.13 & +0.04 & 2 & 0.12 & +0.68 & 2 & 0.20 \\
\hline $\operatorname{Mg}_{\text {I }} \ldots \ldots \ldots$ & +0.42 & 5 & 0.10 & +0.37 & 7 & 0.07 & +0.29 & 7 & 0.08 & +0.17 & 3 & 0.10 & +0.59 & 2 & 0.11 \\
\hline $\mathrm{Al}$ I . ............... & -0.10 & 1 & 0.28 & -0.77 & 1 & 0.28 & -0.72 & 1 & 0.28 & $\ldots$ & & & & & \\
\hline Si I $\ldots \ldots \ldots$ & +0.80 & 2 & 0.27 & +0.84 & 1 & 0.20 & & & & & & & & & \\
\hline $\mathrm{Ca}$ I .............. & +0.22 & 9 & 0.10 & +0.27 & 11 & 0.12 & +0.42 & 8 & 0.11 & +0.03 & 3 & 0.08 & +0.53 & 2 & 0.16 \\
\hline Sc II .......... & +0.02 & 5 & 0.13 & -0.02 & 5 & 0.12 & -0.04 & 3 & 0.13 & & & & & & \\
\hline Ti г ............ & +0.12 & 13 & 0.07 & +0.19 & 10 & 0.09 & +0.15 & 2 & 0.14 & +0.43 & 3 & 0.10 & & & \\
\hline Ti II .......... & +0.43 & 37 & 0.15 & +0.36 & 31 & 0.15 & +0.22 & 13 & 0.15 & +0.60 & 6 & 0.12 & +0.62 & 2 & 0.18 \\
\hline Cr I ............. & -0.38 & 8 & 0.09 & & & & -0.29 & 4 & 0.10 & -0.05 & 4 & 0.08 & +0.22 & 4 & 0.14 \\
\hline $\mathrm{Cr}$ II ............. & +0.12 & 2 & 0.24 & & & & & & & & & & & & \\
\hline $\operatorname{Mn}_{\mathrm{I}} \ldots \ldots \ldots$ & -0.69 & 3 & 0.16 & -0.59 & 2 & 0.17 & -0.38 & 2 & 0.25 & & & & & & \\
\hline $\mathrm{Fe}_{\mathrm{I}} \ldots \ldots \ldots$ & -2.85 & 145 & 0.18 & -2.76 & 161 & 0.18 & -2.72 & 93 & 0.19 & -2.17 & 71 & 0.09 & -2.57 & 30 & 0.23 \\
\hline Fe II ............ & -2.86 & 15 & 0.26 & -2.77 & 12 & 0.25 & -2.71 & 7 & 0.26 & -2.18 & 6 & 0.16 & -2.56 & 4 & 0.28 \\
\hline Co I .............. & -0.08 & 2 & 0.15 & +0.27 & 4 & 0.09 & +0.39 & 3 & 0.09 & & & & & & \\
\hline Ni I ........... & -0.10 & 5 & 0.14 & +0.16 & 6 & 0.07 & -0.25 & 6 & 0.11 & +0.14 & 1 & 0.14 & & & \\
\hline Sr II $\ldots \ldots \ldots$ & -0.37 & 2 & 0.35 & -0.40 & 2 & 0.35 & -0.12 & 2 & 0.31 & & & & & & \\
\hline Y II $\ldots \ldots \ldots \ldots$ & -0.67 & 1 & 0.20 & -0.23 & 2 & 0.16 & +0.16 & 2 & 0.18 & & & & +0.80 & 1 & 0.20 \\
\hline $\operatorname{Zr}$ II $\ldots \ldots \ldots$ & +0.34 & 2 & 0.16 & & & & & & & & & & & & \\
\hline Ва ІІІ............ & -0.57 & 3 & 0.14 & -0.82 & 3 & 0.27 & -0.49 & 4 & 0.17 & -0.58 & 4 & 0.20 & +1.85 & 3 & 0.14 \\
\hline La II .............. & & & & & & & & & & & & & +1.91 & 1 & 0.15 \\
\hline Ce $\mathrm{II} \ldots \ldots \ldots$ & & & & & & & & & & & & & +1.99 & 4 & 0.14 \\
\hline $\operatorname{Pr}$ II $\ldots \ldots \ldots \ldots$ & & & & & & & & & & & & & +1.85 & 3 & 0.14 \\
\hline $\mathrm{Nd}$ II ........ & & & & & & & & & & & & & +1.69 & 21 & 0.12 \\
\hline Eu II .......... & $<-0.5$ & & & $<+0.4$ & & & $<+0.6$ & & & & & & $+1.57:$ & 1 & \\
\hline${ }^{12} \mathrm{C} /{ }^{13} \mathrm{C} \ldots$ & 5 & & & $\sim 20$ & & & $>10$ & & & & & & $\sim 10$ & & \\
\hline Li I $\ldots \ldots \ldots \ldots$ & $<+0.6^{\mathrm{a}}$ & & & $<+1.2^{\mathrm{a}}$ & & & $<+1.2^{\mathrm{a}}$ & & & $<+1.6^{\mathrm{a}}$ & & & $<+1.0:^{\mathrm{a}}$ & & \\
\hline
\end{tabular}

NoTE.- Here $n$ is the number of lines analyzed and SE is the standard error.

${ }^{a}$ The upper limit of $\mathrm{Li}$ is given for $\log \epsilon_{\mathrm{Li}}$ instead of [Li/Fe].

length as the upper limit of the equivalent width. The results are listed in Table 4. The upper limits on the Eu abundances for CS 29502-092 and CS 22877-001 are uninteresting, because the lower limits on $[\mathrm{Ba} / \mathrm{Eu}]$ in these stars are lower than the solar system $r$-process value (e.g., $[\mathrm{Ba} /$ $\mathrm{Eu}]=-0.69$; Arlandini et al. 1999). The lower limit of $[\mathrm{Ba} / \mathrm{Eu}]$ in CS 30314-067 is about -0.1, higher than the solar system $r$-process value, although still much lower than the $s$-process value (e.g., $[\mathrm{Ba} / \mathrm{Eu}]=+1.15$; Arlandini et al. 1999). This suggests that there is a possible contribution of the $s$-process to the heavy elements in this star. We note for completeness that the [Ba/Eu] of CS 22948-027 is about +0.3 , which also implies a significant contribution of the $s$-process to the heavy elements in this object.

The abundance patterns of the other metals seem rather typical for normal (i.e., non carbon- and nitrogen-enhanced) metal-poor stars (McWilliam et al. 1995; Ryan et al. 1996): the $\alpha$-elements $(\mathrm{Mg}, \mathrm{Si}, \mathrm{Ca}$, and $\mathrm{Ti})$ are overabundant by about 0.45 dex on average, while $\mathrm{Cr}$ and $\mathrm{Mn}$ are underabundant by 0.4 dex, and Co is overabundant by $0.19 \mathrm{dex}$.

\subsection{Carbon and Nitrogen}

The carbon abundance was determined by obtaining fits of synthetic spectra of the $\mathrm{CH} A^{2} \Delta-X^{2} \Pi$ band at $4323 \AA$ to the observed ones for CS 30314-067, CS 29502-092, and CS 22877-001. It is also possible to determine the carbon abundance from the $\mathrm{CH} 4315 \AA$ band ( $\mathrm{G}$ band), but the uncertainty would be large due to the difficulty in continuum specification. Therefore we prefer the $\mathrm{CH} 4323 \AA$ band to the $\mathrm{G}$ band for determination of carbon abundance. The same analysis with $C_{2}$ Swan bands at 5165 and $5635 \AA$. was applied to CS 22948-027. We note that our spectrum of CS 30312-100 does not cover the $\mathrm{CH}$ band, and the $\mathrm{C}_{2}$ bands are not detected in the spectrum.

The nitrogen abundances were derived by a similar analysis for the CN band (violet system) at $3880 \AA$ for CS 30314-067, CS 29502-092, and CS 22877-001, and by the standard analysis of the absorption lines of the $\mathrm{CN}$ red system for CS 22948-027. To evaluate the carbon isotope ratio, the lines of the $\mathrm{CH} B^{2} \Sigma^{-}-X^{2} \Pi$ band were used.

The line lists of these molecular bands were produced not only for the abundance analysis, but also to check on the blending of molecular lines, and for the calculation of the effective temperature scale. The $\mathrm{CH}$ and $\mathrm{CN}$ lines used in Aoki et al. (2001) were also applied to this analysis. We also used the lines of the $\mathrm{C}_{2}$ Swan bands and the $\mathrm{CN}$ red system studied by Aoki \& Tsuji (1997a) for the analysis of CS 22948-027. A dissociation energy of $7.75 \mathrm{eV}$ (Aoki \& Tsuji 1997b) was adopted in the analysis of $\mathrm{CN}$ lines. However, there are some suggestions that lower values of the $\mathrm{CN}$ dissociation energy might apply (see references in Aoki \& Tsuji 1997b), and the uncertainty on nitrogen abundances derived from $\mathrm{CN}$ lines is large: the error in nitrogen abundance arising from an error of $0.1 \mathrm{eV}$ in the $\mathrm{CN}$ dissociation energy is estimated to be $0.1-0.15$ dex in our stars. We note, however, that the uncertainty is systematic, and has only a 
small effect on our discussion of the relative abundances between the stars studied in this work. Examples of the spectra at the $\mathrm{CN} 3880 \AA$ band and the $\mathrm{CH} 4323 \AA$ band are shown in Figure 3, along with the synthetic spectra for three different assumed nitrogen and carbon abundances, respectively.

The $\mathrm{C}_{2}$ Swan bands in CS 22948-027 spectrum are shown in Figure 4, along with the synthetic spectra for three different carbon abundances. The carbon abundance determined from the $\mathrm{C}_{2} 5635 \AA$ band was adopted for CS 22948-027 because of the large uncertainty in continuum determination for the $\mathrm{C}_{2} 5165 \AA$ band.

The carbon isotope ratios $\left({ }^{12} \mathrm{C} /{ }^{13} \mathrm{C}\right)$ for CS 30314-067 and CS 29502-092 were determined from the nearly clean $\mathrm{CH}$ lines around $4200 \AA$, adopting the line data used in Aoki et al. (2001). Examples are shown in Figure 5. In the top three panels, we show the observed spectrum of CS 30314-067 and three synthetic ones calculated for ${ }^{12} \mathrm{C} /$ ${ }^{13} \mathrm{C}=3,5$, and 10 , respectively, for the three $\mathrm{CH}$ lines. We find that a ${ }^{12} \mathrm{C} /{ }^{13} \mathrm{C}$ ratio of about 5 is well determined by this analysis. The bottom panels show the spectrum of CS 29502-092, along with the synthetic ones for ${ }^{12} \mathrm{C} /{ }^{13} \mathrm{C}=10$, 20 , and 40 , respectively. In this case, the estimation of the isotope ratio is difficult, because the ${ }^{13} \mathrm{CH}$ lines are much weaker than those in CS 30314-067; hence, a higher S/N spectrum is desirable for accurate determination of the ratio in this star. However, note that the ${ }^{12} \mathrm{C} /{ }^{13} \mathrm{C}$ ratio of CS 29502-092 ( 20) is obviously higher than that of CS 30314067. Only a lower limit $\left({ }^{12} \mathrm{C} /{ }^{13} \mathrm{C}>10\right)$ is estimated for CS 22877-001, because of the low quality of the spectrum. The carbon isotope ratio of CS $22948-027\left({ }^{12} \mathrm{C} /{ }^{13} \mathrm{C} \sim 10\right)$ was determined using $\mathrm{CN}$ lines around $8000 \AA$, following Aoki \& Tsuji (1997a).

The ${ }^{12} \mathrm{C} /{ }^{13} \mathrm{C}$ ratios for CS 29502-092, CS 22948-027, and possibly for CS 22877-001, all fall in the range 10-20, which are typical values for classical $\mathrm{CH}$ stars (Vanture 1992; Aoki \& Tsuji 1997a). These values may reflect carbon production not only by the triple- $\alpha$ reaction, but also from subsequent $\mathrm{CN}$ cycles during the hydrogen-burning phase(s) (Aoki \& Tsuji 1997a). The ${ }^{12} \mathrm{C} /{ }^{13} \mathrm{C}$ of CS 30314067 is substantially lower, near the equilibrium value for the $\mathrm{CN}$ cycle $\left({ }^{12} \mathrm{C} /{ }^{13} \mathrm{C} \sim 3-4\right)$. This result is consistent with the high nitrogen abundance of this object $([\mathrm{N} / \mathrm{Fe}]=+1.2)$, which is also expected from operation of the $\mathrm{CN}$ cycle. This probably indicates that the material in the envelope of this star was strongly affected by hydrogen burning during its
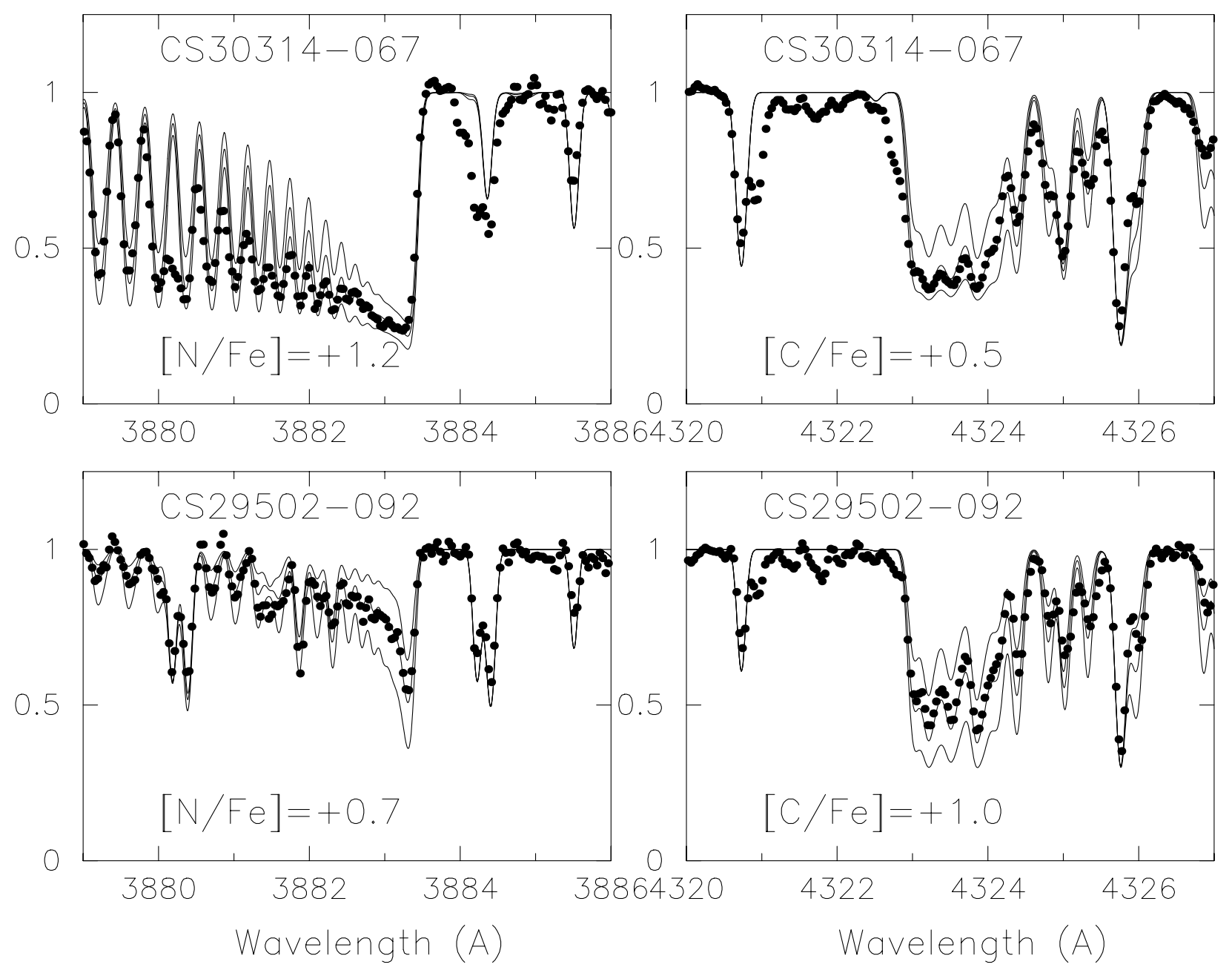

Fig. 3.-Comparison of the observed spectra (filled circles) with the synthetic ones (lines) in the region near the $\mathrm{CN}$ band at $3883 \AA$ and the $\mathrm{CH}$ band at $4323 \AA$ A. On the right the three synthetic spectra differ in steps of $\Delta[\mathrm{C} / \mathrm{Fe}]=0.3$, while on the left the computations were done with the best-fit [C/Fe] determined at $4323 \AA$ and steps of $\Delta[\mathrm{N} / \mathrm{Fe}]=0.3$. The adopted values of $[\mathrm{C} / \mathrm{Fe}]$ and $[\mathrm{N} / \mathrm{Fe}]$, which produce the central synthetic spectrum in each panel, are given in the figures. 

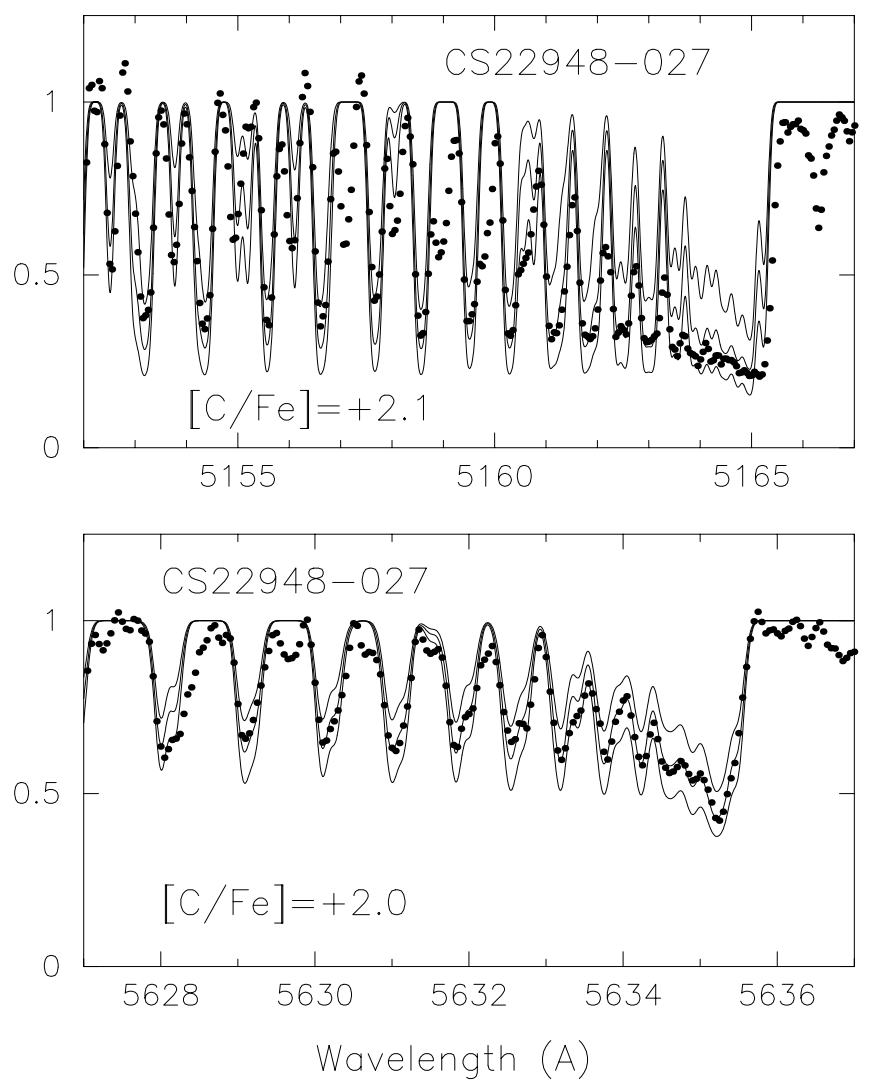

FIG. 4.-Comparison of the observed spectra (filled circles) with the synthetic ones (lines) in the region near the $\mathrm{C}_{2}$ bands at $5160 \AA$ (top) and at $5635 \AA$ (bottom). The computations were done with steps of $\Delta[\mathrm{C} / \mathrm{Fe}]=0.3$.

evolution on the red giant branch. The low derived gravity of this star $(\log g=0.7)$ implies that this object has considerably ascended the red giant branch, and supports the above interpretation.

\subsection{Upper Limits on Lithium Abundance}

A wide spread in $\mathrm{Li}$ abundances is known to exist among carbon-rich, metal-poor stars (e.g., Norris, Ryan, \& Beers 1997a), and Li may be a key element to understanding the formation of these objects. Although most of our stars are giants, and $\mathrm{Li}$ is not expected to currently exist on their stellar surfaces because of destruction from internal mixing processes, it is still worthwhile to search for its presence. We inspected our spectra around the Li $\lambda 6707$ lines, but did not detect any features at the wavelength of Li. We estimated the upper limit on the $\mathrm{Li}$ abundance for these objects assuming $3 \sigma_{W}$ (Table 1) as the upper limit of the equivalent width. Note, however, that Hill et al. (2000) have derived a $\mathrm{Li}$ abundance for CS 22948-027, $\log \epsilon_{\mathrm{Li}}=+0.3$, at a much lower level than our upper limit. It should be pointed out that the derived upper limits for our objects are lower than the primordial $\mathrm{Li}$ abundance, which is reasonable for stars in which internal mixing has affected the surface abundances.

\subsection{Error Estimates}

The errors of the abundance analysis for our stars were estimated following the procedures of Ryan et al. (1996). The errors arising from uncertainties in the atmospheric parameters were evaluated by adding in quadrature the individual errors corresponding to $\Delta T_{\text {eff }}=100 \mathrm{~K}$, $\Delta \log g=0.3$, and $\Delta v_{\text {tur }}=0.5 \mathrm{~km} \mathrm{~s}^{-1}$ for CS 30314-067 (a giant). The errors for $\Delta T_{\text {eff }}$ and $\Delta \log g$ derived for CS 30314-067 were then applied to the other three giants, while the errors derived for LP 625-44 (a subgiant) in Aoki et al. (2000) were applied to the subgiant CS 30312-100. The uncertainty $\Delta v_{\text {tur }}$ was calculated for individual objects. Internal errors were estimated by assuming the errors in the measurement of equivalent widths assessed in $\S 2$, adopting a random error on the $g f$ values of 0.1 dex. The results for the giant CS 30314-067 and the subgiant CS 30312-100 are

TABLE 5

ERror ESTIMATES FOR $[\mathrm{Fe} / \mathrm{H}]$ AND $[\mathrm{X} / \mathrm{Fe}]$

\begin{tabular}{|c|c|c|c|c|c|c|c|c|}
\hline \multirow[b]{2}{*}{ Element } & \multicolumn{4}{|c|}{ CS 30314-067 (Giant) } & \multicolumn{4}{|c|}{ CS 30312-100 (Subgiant) } \\
\hline & $\begin{array}{c}\Delta T_{\text {eff }} \\
(+100 \mathrm{~K})\end{array}$ & $\begin{array}{l}\Delta \log g \\
(+0.3)\end{array}$ & $\begin{array}{c}\Delta v_{\mathrm{tur}} \\
\left(+0.5 \mathrm{~km} \mathrm{~s}^{-1}\right)\end{array}$ & $\sigma_{\text {random }}$ & $\begin{array}{c}\Delta T_{\text {eff }} \\
(+100 \mathrm{~K})\end{array}$ & $\begin{array}{l}\Delta \log g \\
(+0.3)\end{array}$ & $\begin{array}{c}\Delta v_{\text {tur }} \\
\left(+0.5 \mathrm{~km} \mathrm{~s}^{-1}\right)\end{array}$ & $\sigma_{\text {random }}$ \\
\hline $\mathrm{C} \ldots \ldots \ldots$ & +0.00 & -0.06 & +0.09 & 0.14 & & & & \\
\hline $\mathrm{N} \ldots \ldots \ldots$ & +0.00 & -0.03 & +0.09 & 0.22 & & & & \\
\hline $\mathrm{Na}$ I $\ldots . .$. & +0.01 & -0.03 & -0.16 & 0.075 & & & -0.08 & 0.075 \\
\hline $\operatorname{Mg}{ }_{I} \ldots \ldots$ & +0.01 & -0.04 & -0.08 & 0.051 & -0.03 & -0.02 & -0.07 & 0.067 \\
\hline $\mathrm{Al}$ I $\ldots \ldots$. & +0.07 & -0.09 & -0.23 & 0.107 & & & & \\
\hline Si I........ & +0.03 & -0.11 & -0.22 & 0.075 & & & & \\
\hline $\mathrm{Ca} \mathrm{I} \ldots \ldots$ & -0.09 & -0.01 & -0.00 & 0.040 & -0.03 & +0.00 & +0.03 & 0.078 \\
\hline Sc II $\ldots \ldots$ & -0.07 & +0.06 & -0.08 & 0.058 & & & & \\
\hline Ti I ....... & -0.05 & -0.01 & +0.03 & 0.036 & +0.01 & +0.01 & +0.05 & 0.085 \\
\hline Ti II ...... & -0.12 & +0.07 & +0.06 & 0.016 & -0.04 & +0.09 & +0.04 & 0.050 \\
\hline $\mathrm{Cr} \mathrm{I} \ldots \ldots$ & +0.02 & +0.02 & +0.06 & 0.053 & -0.01 & +0.00 & +0.04 & 0.071 \\
\hline $\mathrm{Cr}$ II ...... & -0.18 & +0.11 & +0.07 & 0.105 & & & & \\
\hline Mn I...... & +0.04 & -0.03 & -0.13 & 0.070 & & & & \\
\hline $\mathrm{Fe}$ I........ & +0.15 & -0.04 & -0.09 & 0.008 & +0.09 & -0.01 & -0.07 & 0.013 \\
\hline Fe II ...... & -0.18 & +0.18 & -0.02 & 0.033 & -0.07 & +0.12 & +0.07 & 0.030 \\
\hline Co I ...... & -0.04 & -0.05 & -0.11 & 0.074 & & & & \\
\hline Ni I ....... & +0.00 & -0.03 & -0.13 & 0.060 & +0.03 & -0.02 & -0.01 & 0.135 \\
\hline Sr II $\ldots . .$. & +0.04 & +0.06 & -0.33 & 0.100 & & & & \\
\hline Y II ....... & +0.05 & +0.12 & +0.10 & 0.051 & & & & \\
\hline $\mathrm{Zr}$ II ....... & +0.07 & +0.11 & +0.03 & 0.075 & & & & \\
\hline Ba II ...... & -0.06 & +0.09 & -0.05 & 0.067 & +0.02 & -0.02 & 0.02 & 0.071 \\
\hline
\end{tabular}



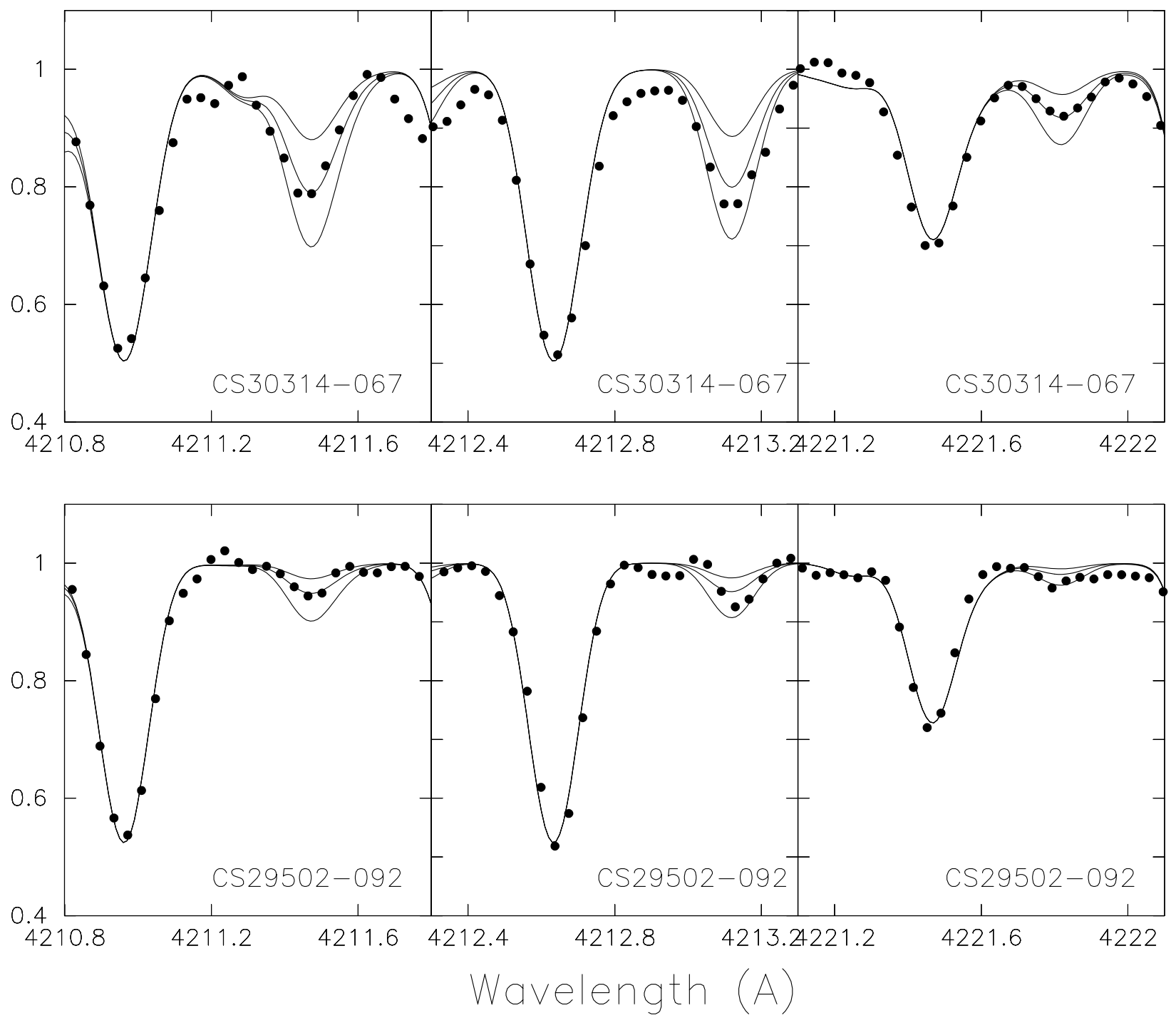

FIG. 5.-Comparison of the observed spectra (filled circles) with the synthetic ones (lines) around $4220 \AA$ A for CS $30314-067$ (top panels) and CS $29502-092$ (bottom panels). The carbon isotope ratios assumed in the computations are ${ }^{12} \mathrm{C} /{ }^{13} \mathrm{C}=3,5$, and 10 for $\mathrm{CS} 30314-067$ and ${ }^{12} \mathrm{C} /{ }^{13} \mathrm{C}=10,20$, and 40 for $\mathrm{CS}$ 29502-092.

given in Table 5 . We note that the errors are provided for the abundance ratio $([\mathrm{X} / \mathrm{Fe}])$, instead of $[\mathrm{X} / \mathrm{H}]$, except for iron. The effect of the uncertainty in atmospheric parameters on an individual elemental abundance $(\Delta[\mathrm{X} / \mathrm{H}])$ sometimes almost cancels out that obtained for the iron abundance $(\Delta[\mathrm{Fe} / \mathrm{H}])$, resulting in a quite small uncertainty in $[\mathrm{X} / \mathrm{Fe}]$. Final estimated abundance uncertainties are included in Table 4.

For carbon and nitrogen abundances determined by spectrum synthesis, we assumed a 0.1 dex fitting error and a 0.1 dex uncertainty in the $g f$ values. The effects of atmospheric parameters are estimated from the analyses assuming the above uncertainties. We estimated the uncertainty of the nitrogen abundance ratio from the $\mathrm{CN}$ band taking into account the uncertainty of carbon abundance derived from the $\mathrm{CH}$ band.

\subsection{Comparison with Previous Work}

One of our objects, CS 22948-027, was studied in detail by Hill et al. (2000). Our results for iron, carbon, and nitro- gen abundances agree with their values within the derived uncertainties. The ${ }^{12} \mathrm{C} /{ }^{13} \mathrm{C}$ ratio also agrees rather well with their result, and the large overabundances of $\mathrm{Na}$ and $\mathrm{Mg}$ shown by their work are also confirmed. The large overabundances of neutron-capture elements for this star are also clearly supported by the present work. However, note that there is a systematic difference in the abundances of heavy elements $(Z \geq 39)$; our analysis obtains $0.2-0.5$ dex smaller abundances. One reason for this discrepancy is the difference in atmospheric parameters adopted in the two analyses. Our effective temperature and gravity $(\log g)$ are lower by $200 \mathrm{~K}$ and 0.8 dex, respectively, than those of Hill et al. (2000). The abundance ratios of these heavy elements to iron are not so sensitive to the effective temperature assumed, but they are rather sensitive to the adopted gravity. The abundance of $\mathrm{Ba}$ increases with increasing $\log g$, while the $\mathrm{Fe}$ abundance derived from $\mathrm{Fe} \mathrm{I}$ lines is insensitive to $\log g$. Our lower $\log g$ results in smaller abundance ratios of $\mathrm{Ba}$ and the other neutron-capture elements compared with those in Hill et al. (2000). Our larger micro- 
turbulent velocity also results in lower abundances when strong lines (e.g., Ba II lines in CS 22948-027) are used in the analysis.

The elemental abundances of CS 22948-027 derived by our analysis agree rather well with the results of Preston \& Sneden (2001), who adopted similar values of atmospheric parameters $\left(T_{\text {eff }}=4600 \mathrm{~K}, \log g=0.8, v_{\text {tur }}=2.3 \mathrm{~km} \mathrm{~s}^{-1}\right)$ for this star. This result suggests that the differences between the abundances derived by Hill et al. (2000) and those derived by this work and by Preston $\&$ Sneden (2001) are mainly due to the atmospheric parameters adopted.

\section{DISCUSSION}

\subsection{Abundance Ratios of Carbon and the Neutron-Capture Elements}

Figure 6 shows $[\mathrm{C} / \mathrm{Fe}]$ as a function of $[\mathrm{Fe} / \mathrm{H}]$ for the carbon-rich, metal-poor stars studied in the present work, along with those for other similarly carbon rich stars described in the literature (McWilliam et al. 1995; Norris et al. 1997a, 1997b, 2001; Bonifacio, Centurion, \& Molaro 1999; Hill et al. 2000; Aoki et al. 2001). For comparison, carbon abundances of disk stars and other metal-poor stars are also shown (Ryan, Norris, \& Bessell 1991; Tomkin et al. 1992, 1995; McWilliam et al. 1995; Gustafsson et al. 1999). While the metal-rich (disk) stars are dwarfs, the metal-poor stars include giants that may have been affected by the $\mathrm{CN}(\mathrm{O})$ cycle and internal mixing in the objects themselves (e.g., by first dredge-up). Therefore, it remains possible that the original carbon abundances for the most metal deficient stars may have been higher than those shown in the figure.

Figure 6 clearly illustrates the large overabundances of carbon, by more than $1 \mathrm{dex}$, for three of the stars in the present program. The excess of carbon in a fourth star, CS $30314-067$, is not distinctive $([\mathrm{C} / \mathrm{Fe}]=+0.5)$, but the nitrogen overabundance is large in this star, and hence the $[\mathrm{C}+\mathrm{N} / \mathrm{Fe}]$ of this star is comparable with those of CS 29502-092 and CS 22877-001 (see Table 4). Since CS 30314067 has a lower effective temperature and surface gravity than the other stars, the mixing of material affected by the $\mathrm{CN}$ cycle would tend to reduce $[\mathrm{C} / \mathrm{Fe}]$ and increase $[\mathrm{N} / \mathrm{Fe}]$ in the stellar surface. Hence we treat CS 30314-067 as a "carbon-rich" star similar to CS 29502-092 and CS 22887001. Our analysis confirms the extremely large overabundance of carbon in CS 22948-027, as found by Hill et al. (2000). There is a large variation in $[\mathrm{C} / \mathrm{Fe}]$ for stars around $[\mathrm{Fe} / \mathrm{H}] \sim-3$, and there seems to be a gap between the very carbon rich stars $([\mathrm{C} / \mathrm{Fe}] \sim 2)$ and the mildly carbon enhanced stars $([\mathrm{C} / \mathrm{Fe}] \sim 1)$, including the newly studied objects here, as pointed out by Norris, Ryan, \& Beers (1997a).

In Figure 7 we show derived $[\mathrm{Ba} / \mathrm{Fe}]$, as a function of $[\mathrm{Fe} / \mathrm{H}]$, for our carbon-rich stars, together with other metal-poor stars from the literature. Carbon-rich stars $([\mathrm{C} /$ $\mathrm{Fe}] \geq 0.5$ ) are shown by filled symbols. The result for CS 22948-027 confirms the large overabundance of barium found by Hill et al. (2000). Four additional stars shown (LP 625-44, LP 706-7, CS 22898-027, and CS 29497-034) exhibit similar overabundances of barium $([\mathrm{Ba} / \mathrm{Fe}] \gtrsim 2)$.

The other four stars in our sample (besides CS 22948-027) exhibit no excess of barium. It is known that the barium abundance ratio $([\mathrm{Ba} / \mathrm{Fe}])$ in metal-poor stars is usually lower than the solar value (i.e., $[\mathrm{Ba} / \mathrm{Fe}]<0$ ). This is often interpreted as a result of the increasing contribution of the $s$-process to the composition of more metal rich stars (Spite \& Spite 1978; Truran 1981). The abundance ratios of Ba to

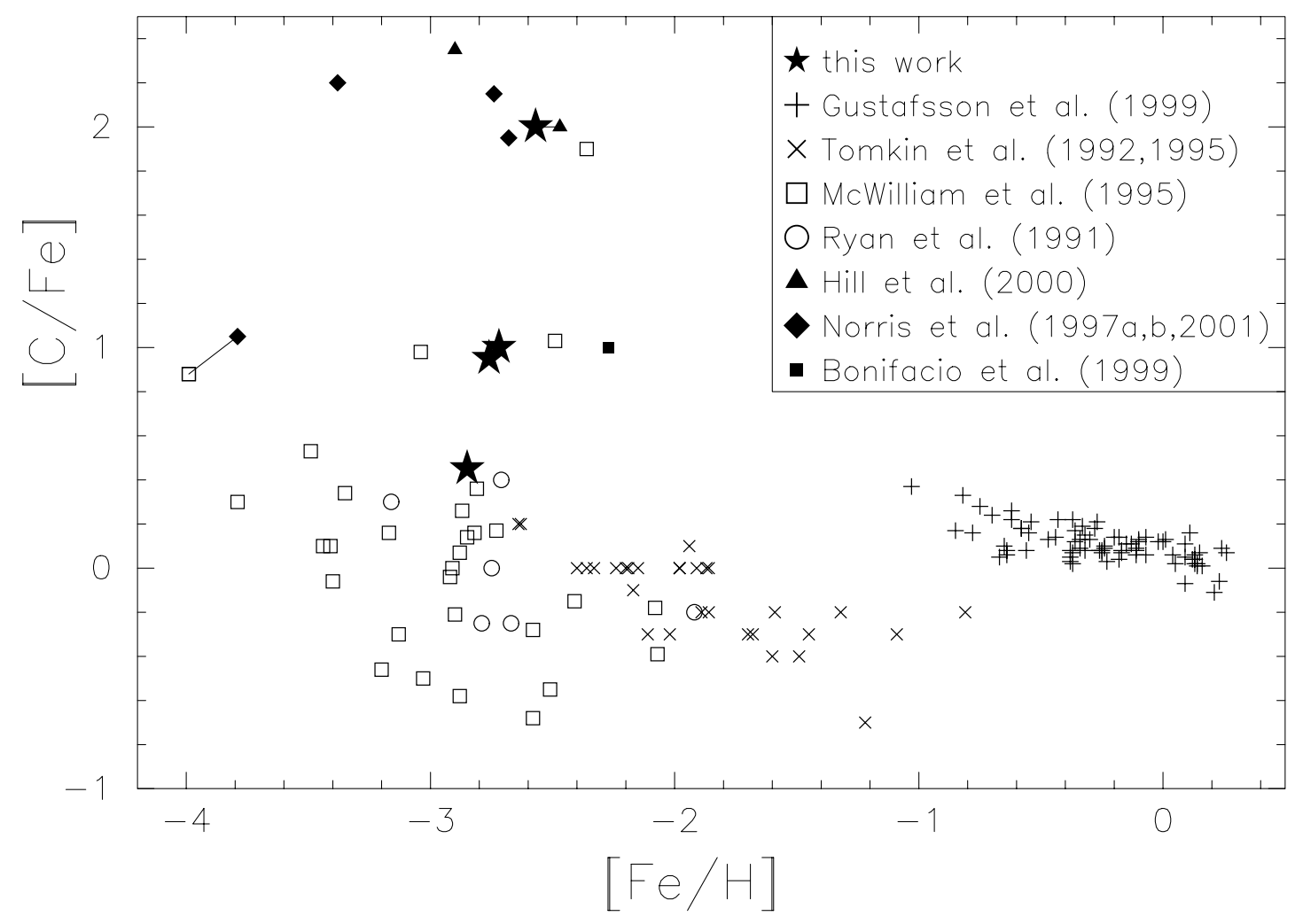

Fig. 6. - Carbon abundance ratios $([\mathrm{C} / \mathrm{Fe}]$ ) as a function of $[\mathrm{Fe} / \mathrm{H}]$. Present results are shown by stars, and compared with results from other studies (see text). 


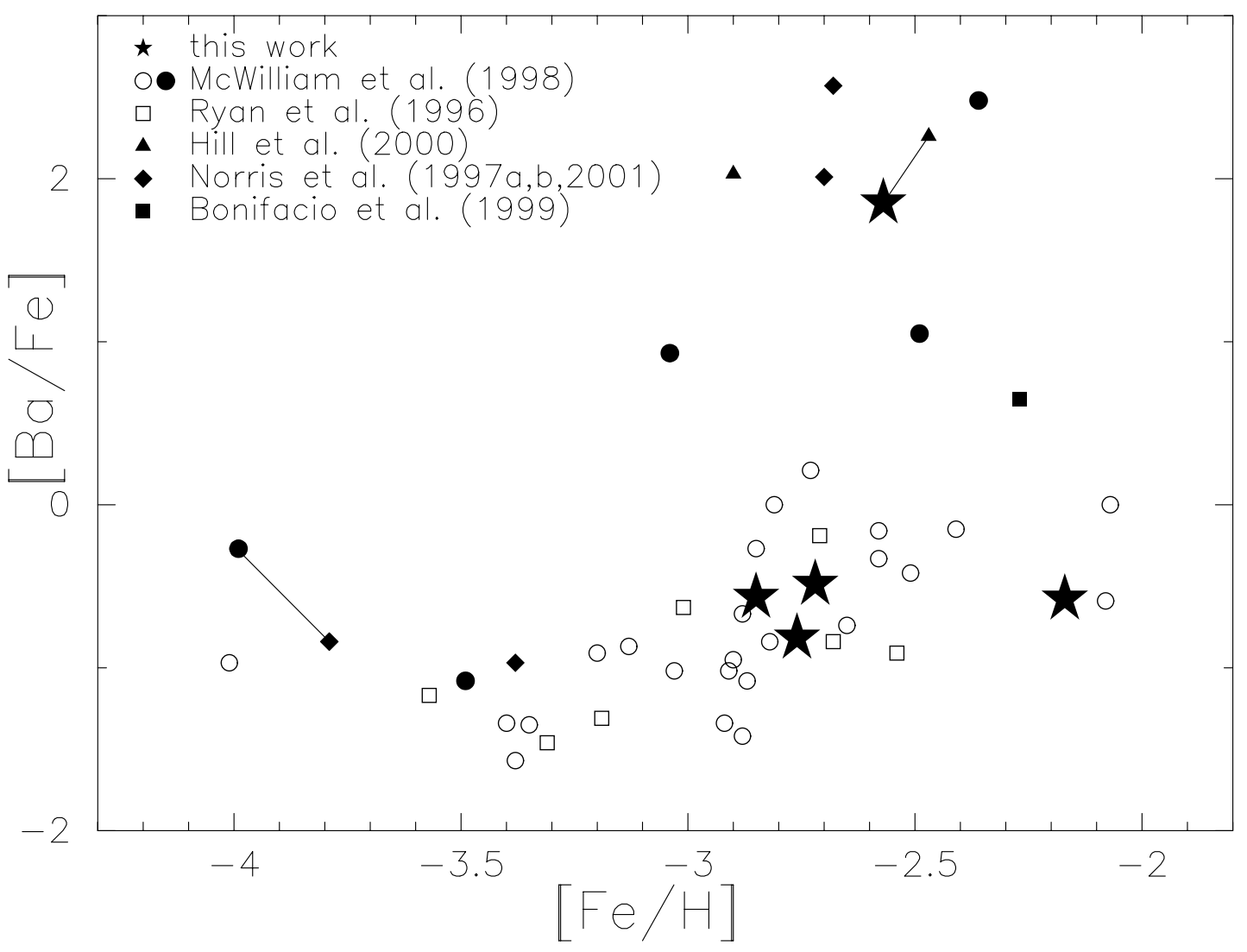

Fig. 7.-Present results for barium abundances $([\mathrm{Ba} / \mathrm{Fe}])$ are shown by stars. The barium abundances of metal-poor stars derived by other studies are also shown. The line connecting two symbols is for the star CS 22948-027, also studied by Hill et al. (2000).

Eu for metal-poor stars studied by McWilliam (1998) suggest that the $\mathrm{Ba}$ originates in the $r$-process, instead of the $s$-process, for most objects with $[\mathrm{Fe} / \mathrm{H}]<-2.4$. From Figure 7, it is clear that the Ba abundances of our four stars lie on the general trend of Ba abundances in this metallicity range. This fact implies that there was likely to have been almost no contribution of the s-process to the $\mathrm{Ba}$ abundances in these carbon-rich stars. In addition to the Ba result, these stars also do not exhibit overabundances of $\mathrm{Sr}$ and $\mathrm{Y}$. We note that the lower limit of $[\mathrm{Ba} / \mathrm{Eu}]$ in $\mathrm{CS}$ 30314-067 is higher than the solar system $r$-process value (§ 4.1), and some additional contribution to heavy elements seems to exist in this object. Although this could be due to a nonuniversal $r$-process, this star also has a low ${ }^{12} \mathrm{C} /{ }^{13} \mathrm{C}$ ratio, and the excess of the $\mathrm{Ba} / \mathrm{Eu}$ ratio may indicate a contribution from the $s$-process.

\subsection{Carbon-Rich Stars without Excess of Neutron-Capture Elements}

The result of this study is summarized in Figure 8 , in which $[\mathrm{Ba} / \mathrm{Fe}]$ is shown as a function of the total abundance ratios of carbon and nitrogen $([\mathrm{C}+\mathrm{N} / \mathrm{Fe}])$. In addition to the abundances of the objects shown in Figures 6 and 7 , some moderately nitrogen rich, Ba-rich objects are also shown in the figure (HD 25329 and HD 74000 from Beveridge \& Sneden 1994, and LP 685-47 from Ryan et al. 1991). This figure also includes the nitrogen-rich star (CS 22949-037) studied by Norris et al. (2001). Values of $[\mathrm{C}+\mathrm{N} / \mathrm{Fe}]$ and $[\mathrm{Ba} / \mathrm{Fe}]$ typical of "normal" metal-poor stars are denoted by the cross at the lower left corner of the

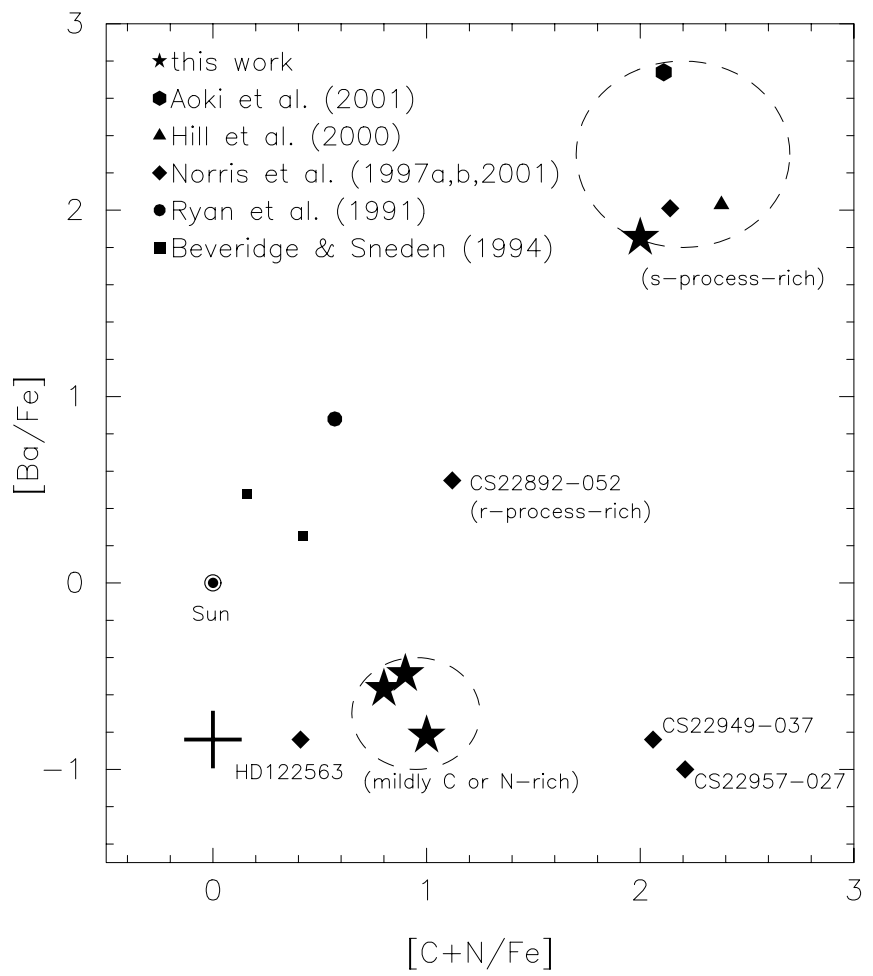

FIG. 8.-Barium abundances as a function of the total abundance ratios of carbon and nitrogen $([(\mathrm{C}+\mathrm{N}) / \mathrm{Fe}])$ for carbon- or nitrogen-rich stars. The abundances of HD 122563, which is not a carbon-rich star, are displayed for comparison. 
figure. The value of $[\mathrm{Ba} / \mathrm{Fe}]=-0.84$ was adopted from the mean of the $\mathrm{Ba}$ abundances of $[\mathrm{Fe} / \mathrm{H}]<-2.5$ objects in McWilliam (1998) (excluding the $r$-process-rich star CS 22892-052). The [C + N/Fe] value of "normal" metal-poor stars is not well established, and there appears to be a rather large scatter. Here we simply assume the solar abundance ratio $[\mathrm{C}+\mathrm{N} / \mathrm{Fe}]=0$.

The present work indicates that three of our mildly carbon- and/or nitrogen-rich $([\mathrm{C}+\mathrm{N} / \mathrm{Fe}] \sim+1)$ stars exhibit no excess of neutron-capture elements. This result is difficult to explain by the scenario of mass transfer from thermally pulsing AGB stars (which probably applies to CS 22948-027), and requires another process of carbon enrichment that does not affect the neutron-capture elements. The carbon and nitrogen overabundances in the stars with normal neutron-capture elements are moderate, in contrast to the very large carbon overabundance in the s-processrich stars like CS 22948-027 and LP 625-44 (Aoki et al. 2001). However, there exist at least two extremely carbon and nitrogen rich stars with normal neutron-capture element abundances for their metallicity, CS 22957-027 (Norris et al. 1997b) and CS 22949-037 (Norris et al. 2001). There also exists a moderately carbon rich and s-processrich star, CS 29529-012 (Bonifacio et al. 1999). The three moderately nitrogen rich, Ba-rich objects shown in Figure 6 may also have an s-process-rich nature, as suggested by Beveridge \& Sneden (1994).

Discussion of the absolute value of elemental overabundances for the objects under study is made difficult because, for a given star, it depends on the assumed dilution due to mixing in the stellar envelope. It may also depend on the mass accreted from the companion (and the mixing in the donor star), if the overabundance is caused by mass transfer across a binary system. We simply point out the importance of the study of chemical composition and binarity for the class of "mildly carbon rich" stars, as well as for "extremely carbon rich" objects.

Norris et al. (1997b) discussed the carbon (and nitrogen) enrichment in zero-metallicity, low-mass stars studied by Hollowell, Iben, \& Fujimoto (1990) as a candidate for the explanation of the properties of the carbon-rich, neutroncapture-element-poor star CS 22957-027. The theoretical study of nucleosynthesis and mixing processes in such stars has been recently extended to other metallicity and mass ranges by Fujimoto, Ikeda, \& Iben (2000), who investigated the possibility of carbon and nitrogen overproduction during He core flash or AGB evolution. They showed that the low-mass $\left(M \lesssim 1 M_{\odot}\right)$ stars with $-4<[\mathrm{Fe} / \mathrm{H}]$ $<(-3 \sim-2)$ do not become carbon rich by core flash at the tip of the red giant branch, but rather through He shell flashes near the base of the AGB. An important prediction of this study is that, while the enrichment of s-process elements occurs in higher mass $\left(M \gtrsim 1 M_{\odot}\right)$ stars by the third dredge-up (their case $\mathrm{II}^{\prime}$ ), such enrichment does not occur in low-mass stars (their case II). Our new results may be examples of this scenario.

Our present expectation is that an $s$-process-rich, carbon-enhanced, metal-poor star is likely to be the companion of a higher mass star from which neutron-captureelement-rich material has been accreted. In contrast, a carbon-rich metal-poor star with normal neutron-capture element abundances would be a low-mass $\left(M \sim 0.8 M_{\odot}\right)$ star in which carbon and nitrogen have been self-enhanced during its AGB evolution without affecting the abundances of its neutron-capture elements. This latter, "intrinsic" scenario would require that we are currently seeing such stars during their brief lifetime on the AGB. An alternative possibility is that these stars are companions of slightly higher mass $\left(0.8<M \lesssim 1 M_{\odot}\right)$ stars from which carbon-rich material without excess of neutron-capture elements has been accreted. It should be noted that the binarity of the carbon-rich, neutron-capture-element-poor star CS 22957027 was recently confirmed by Preston \& Sneden (2001). It is clearly of great importance to search for binarity among other similar stars.

Fujimoto et al. (2000) also predicted overabundances of nitrogen due to hydrogen mixing following the helium-shell flash in low-mass, low- metallicity stars. Two of our stars with normal neutron-capture-element abundances exhibit large nitrogen abundances (CS 30314-067 and CS 29502092, $[\mathrm{N} / \mathrm{H}] \sim+1)$, but one does not (CS 22877-001, [N/ $\mathrm{H}] \sim 0$ ). As mentioned in $\S 4.2$, the systematic uncertainty of the nitrogen abundance derived from the $\mathrm{CN}$ molecular band is large, but the relative abundances among our stars should be reliable. A variation of nitrogen abundances certainly exists among our mildly carbon-rich stars with similar metallicity $([\mathrm{Fe} / \mathrm{H}] \sim-2.8)$. This result provides a constraint on the evolutionary models of very metal poor AGB stars.

The above discussion concerning carbon and nitrogen production by low-mass metal-poor stars is still quite primitive, and further tests of consistency between observation and models are indispensable. We also need to consider whether other possible processes that enrich carbon at very low metallicity exist. There is no attractive candidate at present, but we should recall the discussion by Norris et al. (1997a) concerning the possibility of large amounts of carbon and nitrogen ejection from "hypernovae" of very high mass in the early era of the Galaxy. As yet there has been no quantitative analysis of the role of mass loss in driving freshly synthesized $\mathrm{C}$ and/or $\mathrm{N}$ into the interstellar medium from the surface of high-mass, low-metallicity stars. If such stars eventually collapse to black holes as failed supernovae, but have already expelled significant quantities of $\mathrm{C}$ or $\mathrm{N}$, they would enrich the early protogalaxy in these elements without also yielding heavier metals. Such objects could contribute to the high $[\mathrm{C} / \mathrm{Fe}]$ and $[\mathrm{N} / \mathrm{Fe}]$ ratios observed in some of the oldest objects. A challenge for this mechanism is that stellar winds are reduced in low-metallicity environments, but massive stars that not only attain high luminosities but also synthesize their own $\mathrm{C}$ and/or $\mathrm{N}$ may overcome this potential problem. We hope that this mechanism will be explored quantitatively in coming years.

\subsection{Carbon Enrichment in the r-Process-Enhanced Star CS 22892-052}

The discovery of the extremely $r$-process enhanced metalpoor star CS 22892-052 has had a major impact on the study of neutron-capture nucleosynthesis and cosmochronology using the radioactive element $\mathrm{Th}$. The excess of carbon in this object $([\mathrm{C} / \mathrm{Fe}] \sim+1.0)$ has influenced the subsequent discussion concerning the site of the $r$-process that produced the abundance pattern in this star, and hence is worthy of consideration in some detail.

Figure 8 shows that CS 22892-052 is also mildly carbon rich (and nitrogen-rich), similar to three of our stars. Our mildly carbon rich stars have a quite "normal" abundance 
pattern except for carbon and nitrogen, in contrast to $s$ process-rich stars like LP 625-44 (Aoki et al. 2000). If another process provides CS 22892-052 with $r$-process-rich material, an almost pure $r$-process abundance pattern will be observed in the object. Mildly carbon rich stars seem common among very metal poor giants. It is possible that an independent, infrequent process must be invoked to produce the $r$-process element overabundances even in stars with carbon and nitrogen excesses like CS 22892-052. One possibility is the "peppering" of the surface of CS 22892052 by the nucleosynthesis products from a massive companion that underwent a Type II supernova explosion, and may now be present as a massive collapsed object, such as a black hole (Qian \& Wasserburg 2001). The suggestion by Preston \& Sneden (2001) that CS 22892-052 may exhibit an anomalously short period $(\sim 180$ days $)$ might also be expected in such a scenario. Clearly, this period must be confirmed by additional data, and searches for periodicity in the other known extremely $r$-process enhanced metalpoor star, CS 31082-001 (Cayrel et al. 2001), should be undertaken. In any event, the lack of neutron-capture element enhancement in our mildly carbon rich stars certainly suggests that the origin of $r$-process element enhancement in CS 22892-052 might be independent of the process responsible for its carbon and nitrogen excesses. It should be noted that neither the moderately $r$-process rich star HD 115444 (Westin et al. 2000) nor the extremely $r$-process rich star CS 31082-001 exhibit carbon enhancement (Cayrel et al. 2001).

\subsection{Isotope Ratios of Carbon and Nitrogen}

The species ${ }^{13} \mathrm{C}$ and ${ }^{14} \mathrm{~N}$ are both produced from ${ }^{12} \mathrm{C}$ via hydrogen burning in the $\mathrm{CN}$ cycle; hence, the measurement of ${ }^{13} \mathrm{C}$ and $\mathrm{N}$ in several of our stars permits us to compare the isotope ratios to those expected. Although we do not have isotope information for $\mathrm{N}$, it is reasonable to assume that the dominant isotope is ${ }^{14} \mathrm{~N}$.

When the $\mathrm{CN}$ cycle runs in equilibrium, the isotope ratios attain values of ${ }^{12} \mathrm{C} /{ }^{13} \mathrm{C} \simeq 3$ and ${ }^{12} \mathrm{C} /{ }^{14} \mathrm{~N} \simeq 0.03$, equivalent to ${ }^{13} \mathrm{C} /{ }^{14} \mathrm{~N} \simeq 0.01$ (Arnould, Goriely, \& Jorissen 1999). When we measure ${ }^{12} \mathrm{C}$, we do not know what fraction has passed through the $\mathrm{CN}$ cycle, so the isotope ratios involving ${ }^{12} \mathrm{C}$ are difficult to interpret. However, since both ${ }^{13} \mathrm{C}$ and ${ }^{14} \mathrm{~N}$ are believed to be produced only by hydrogen burning in the $\mathrm{CN}$ cycle sequence, their isotope ratios should reflect the degree of processing.

In Table 6 we show the element and isotope ratios for the four stars in this paper with either measured ${ }^{13} \mathrm{C}$ values or limits, and the two stars studied by Norris et al. (1997a, 1997b) and Aoki et al. (2001). It is immediately clear that the ${ }^{13} \mathrm{C} /{ }^{14} \mathrm{~N}$ ratio is well in excess of the $\mathrm{CN}$ cycle equilibrium value. That is, even when ${ }^{12} \mathrm{C}$ is hydrogen burning to ${ }^{13} \mathrm{C}$, it is not being burnt on to ${ }^{14} \mathrm{~N}$ in the equilibrium ratio. It is perhaps surprising that this is the case even in the star with ${ }^{12} \mathrm{C} /{ }^{13} \mathrm{C}=5$.

The nonequilibrium isotope ratios should help identify the sites in which such large carbon and nitrogen excesses were produced. Normally, the $\mathrm{CN}$ cycle reaches equilibrium on a short timescale, after a few proton-capture lifetimes, equivalent to the consumption of typically one proton per initial nucleus (e.g., Clayton 1968). However, one case in which high ${ }^{13} \mathrm{C} /{ }^{14} \mathrm{~N}$ ratios might be achieved is if the star's evolutionary timescale during $\mathrm{CN}$ cycling is shorter than the timescale for the ${ }^{13} \mathrm{C}(p, \gamma){ }^{14} \mathrm{~N}$ reaction, so conditions become unfavorable for hydrogen burning before ${ }^{14} \mathrm{~N}$ production completes. This might imply that the hydrogen burning occurred during more rapid stages of evolution, on the giant branch or during short-lived thermal pulses on the $\mathrm{AGB}$, rather than on the main sequence. Truncation of the $\mathrm{CN}$ cycle could have happened if the temperature fell below that required for hydrogen burning, or if protons were in short supply, as might happen in the $\mathrm{He}$ intershell of a thermally pulsing AGB star. The latter possibility is underlined by the analysis of the very metal poor binary LP 625-44: the ${ }^{13} \mathrm{C}$ pocket of its AGB component, produced by protons mixed down from the H-rich envelope, was only $1 / 24$ that expected from higher metallicity models (Ryan et al. 2001). Proton starvation could leave a star unable to burn beyond ${ }^{13} \mathrm{C}$, and lead to a truncated $\mathrm{CN}$ cycle.

It was noted above that a high, nonequilibrium ${ }^{13} \mathrm{C} /{ }^{14} \mathrm{~N}$ ratio exists even for the star with the low, equilibrium value of ${ }^{12} \mathrm{C} /{ }^{13} \mathrm{C}=5$. However, this may nevertheless be compatible with a truncated CN cycle. Clayton (1968, his Fig. 5-15) shows the approach to equilibrium for burning at $20 \times 10^{6}$ $K$. At a stage when $0.8-0.9$ protons have been consumed per initial nucleus, before the cycle reaches equilibrium, ${ }^{13} \mathrm{C} /$ ${ }^{14} \mathrm{~N} \sim 0.5$, but already ${ }^{12} \mathrm{C} /{ }^{13} \mathrm{C} \simeq 4 ;{ }^{13} \mathrm{C}$ and ${ }^{12} \mathrm{C}$ come into equilibrium with each other faster than with ${ }^{14} \mathrm{~N}$. ${ }^{13} \mathrm{C} /{ }^{14} \mathrm{~N}$ then decreases as equilibrium is approached, but with little change in ${ }^{12} \mathrm{C} /{ }^{13} \mathrm{C}$. Hence, the apparent contradiction between equilibrium ${ }^{12} \mathrm{C} /{ }^{13} \mathrm{C}$ ratios and nonequilibrium ${ }^{13} \mathrm{C} /{ }^{14} \mathrm{~N}$ ratios may be a pointer to the time at which proton truncation occurred, as well as to the mean number of proton captures. The timescale for the $\mathrm{CN}$ cycle to reach this stage in Clayton's example was $\sim 10^{4} \mathrm{yr}$, of the same order as the interval between the thermal pulses of an AGB star. Furthermore, the equilibrium ${ }^{13} \mathrm{C} /{ }^{14} \mathrm{~N}$ ratio is temperature dependent, and would be higher for $T>20 \times 10^{6}$ $\mathrm{K}$, making it even easier to avoid overproduction of ${ }^{14} \mathrm{~N}$ relative to ${ }^{13} \mathrm{C}$ in hotter environments such as an $\mathrm{AGB}$ star's He intershell. We would welcome a more thorough testing of these possibilities in AGB and other stellar

TABLE 6

ISOTOPE RATIOS OF CARBON AND NITROGEN

\begin{tabular}{|c|c|c|c|c|c|c|c|}
\hline Star & {$[\mathrm{Fe} / \mathrm{H}]$} & {$[\mathrm{C} / \mathrm{Fe}]$} & {$[\mathrm{N} / \mathrm{Fe}]$} & ${ }^{12} \mathrm{C} /{ }^{13} \mathrm{C}$ & ${ }^{12} \mathrm{C} / \mathrm{N}$ & ${ }^{13} \mathrm{C} / \mathrm{N}$ & Reference \\
\hline CS $30314-067 \ldots \ldots$ & -2.85 & 0.5 & 1.2 & 5 & 0.63 & 0.13 & 1 \\
\hline CS $29502-092 \ldots \ldots$. & -2.76 & 1.0 & 0.7 & 20 & 7.2 & 0.36 & 1 \\
\hline CS $22948-027 \ldots \ldots$ & -2.57 & 2.0 & 1.8 & 10 & 5.5 & 0.55 & 1 \\
\hline CS $22877-001 \ldots \ldots$ & -2.17 & 1.0 & 0.0 & $>10$ & 36 & $<3.5$ & 1 \\
\hline CS $22957-027 \ldots \ldots$ & -3.38 & 2.2 & 2.0 & 10 & 5.5 & 0.55 & 2 \\
\hline LP $625-44 \ldots \ldots \ldots$ & -2.68 & 1.95 & 1.65 & 20 & 7.2 & 0.36 & 3,4 \\
\hline
\end{tabular}

REFERENCES.- (1) This work; (2) Norris et al. 1997b; (3) Norris et al. 1997a; (4) Aoki et al. 2001. 
models, to see whether models can be identified that possess the conditions required to reproduce the observed ratios.

\section{SUMMARY}

Our abundance analysis for very metal poor stars with strong $\mathrm{CH}$ bands, based on high-resolution spectra, revealed that four of our five program stars do not exhibit overabundances of barium $([\mathrm{Ba} / \mathrm{Fe}]<0)$. Three of the four objects without $\mathrm{Ba}$ excesses have mildly enhanced carbon and/or nitrogen $([\mathrm{C}+\mathrm{N}] \sim+1)$. Prior to this work, only one carbon-rich, neutron-capture-element-poor star was known (CS 22957-027). Our study indicates that this kind of object is not uncommon among very metal deficient stars.

We have discussed possible processes to explain the chemical nature of carbon-rich, metal-poor stars. One candidate is the helium-shell flash near the base of the AGB in low-mass $\left(M \lesssim 1 M_{\odot}\right)$ stars proposed by Fujimoto et al. (2000). They showed that carbon and nitrogen enrichment occurs by this process in stars with $-4 \lesssim[\mathrm{Fe} / \mathrm{H}] \lesssim-3$ $(\sim-2)$ stars, but the $s$-process elements are enhanced by the third dredge-up only in higher mass $\left(M \gtrsim 1 M_{\odot}\right)$ stars. Our result can be interpreted within this framework, but a further test of consistency between observations and the models, such as for nitrogen abundances, is required.

The fact that mildly carbon rich stars with normal neutron-capture element abundances are not rare among metal-poor giants suggests that the moderate over- abundance of carbon $([\mathrm{C} / \mathrm{Fe}] \sim 1)$ in the famous $r$-processenhanced star CS 22892-052 may have originated in the same process that produced our carbon-rich stars. This implies a decoupling of carbon and $r$-process production processes for this object.

Carbon-enhanced stars are quite numerous among very metal deficient stars. Nevertheless, among the limited number of such stars studied so far, a large variation in the abundances of carbon and neutron-capture elements has been found. To understand the nature of this important class of objects, and their role in the early Galaxy, detailed abundance studies for carbon-rich objects covering a wider metallicity range is desirable. Long-term studies to detect, and quantify, the existence of binarity among such stars are crucial as well.

The authors are grateful to the Australian Time Allocation Committee for their continued support for our studies of the most metal deficient stars and to the Director and staff of the Anglo-Australian Observatory for providing the facilities for this study. The authors wish to thank John Lattanzio and M. Fujimoto for helpful discussions. Thanks are also due to the anonymous referee for useful comments. T. C. B. acknowledges partial support from NSF grant AST 00-98549. S. G. R acknowledges support from PPARC grant PPA/O/S/1998/00658.

\section{REFERENCES}

Abia, C., Domínguez, I., Straniero, O., Limongi, M., Chieffi, A., \& Isern, J. 2001, ApJ, 557, 126

Alonso, A., Arribas, S., \& Martínez-Roger, C. 1999, A\&AS, 140, 261

Aoki, W., Norris, J. E., Ryan, S. G., Beers, T. C., \& Ando, H. 2000, ApJ, 536, L97

Aoki, W., Ryan S. G., Norris, J. E., Beers, T. C., Ando, H., Iwamoto, N., Kajino, T., Mathews, G. J., \& Fujimoto, M. Y. 2001, ApJ, 561, 346

Aoki, W., \& Tsuji, T. 1997a, A\&A, 317, 845

1997b, A\&A, 328, 175

Arlandini, C., Käppeler, F., Wisshak, K., Gallino, R., Lugaro, M., Busso, M., \& Straniero, O. 1999, ApJ, 525, 886

Arnould, M., Goriely, S., \& Jorissen, A. 1999, A\&A, 347, 572

Bard, A., \& Kock, M. 1994, A\&A, 282, 1014

Bard, A., Kock, A., \& Kock, M. 1991, A\&A, 248, 315

Beers, T. C. 1999, in ASP Conf. Ser. 165, Third Stromlo Meeting, ed. B. Gibson, T. Axelrod, \& M. Putman (San Francisco: ASP), 206

Beers, T. C., Preston, G. W., \& Shectman, S. A. 1985, AJ, 90, 2089 1992, AJ, 103, 1987

Bessell, M. S. 1990, PASP, 102, 1181

Bessell, M. S., Castelli, F., \& Plez, B. 1998, A\&A, 333, 231

Beveridge, C. R., \& Sneden, C. 1994, AJ, 108, 285

Biémont, E., Karner, C., Meyer, G., Träger, F., \& zu Putlitz, G. 1982, A\&A, 107,166

Bizzarri, A., Huber, M. C. E., Noels, A., Grevesse, N., Bergeson, S. D., Tsekeris, P., \& Lawler, J. E. 1993, A\&A, 273, 707

Blackwell, D. E., Booth, A. J., Petford, A. D., \& Laming, J. M. 1989, MNRAS, 236, 235

Blackwell, D. E., Ibbetson, P. A., Petford, A. D., \& Shallis, M. J. 1979a, MNRAS, 186, 633

Blackwell, D. E., Menon, S. L. R., Petford, A. D., \& Shallis, M. J. 1982a, MNRAS, 201, 611

Blackwell, D. E., Petford, A. D., \& Shallis, M. J. 1979b, MNRAS, 186, 657

Blackwell, D. E., Petford, A. D., Shallis, M. J., \& Leggett, S. 1982c, MNRAS, 199, 21

Blackwell, D. E., Petford, A. D., Shallis, M. J., \& Simmons, G. J. 1980, MNRAS, 191, 445 1982d, MNRAS, 199, 43

Blackwell, D. E., Petford, A. D., \& Simmons, G. J. 1982b, MNRAS, 201, 595

Bond, H. E. 1974, ApJ, 194, 95

Bord, D. J., Barisciano, L. P., \& Cowley, C. R. 1996, MNRAS, 278, 997

Bonifacio, P.. Centurion, M., \& Molaro, P. 1999, MNRAS, 309, 533

Bonifiacio, P., Molaro, P., Beers, T. C., \& Vladilo, G. 1998, A\&A, 332, 672

Booth, A. J., Blackwell, D. E., Petford, A. D., \& Shallis, M. J. 1984, MNRAS, 208, 147

Busso, M., Gallino, R., \& Wasserburg, G. J. 1999, ARA\&A, 37, 239
Cardon, B. L., Smith, P. L., Scalo, J. M., Testerman, L., \& Whaling, W. 1982, ApJ, 260, 395

Cayrel, R., et al. 2001, Nature, 409, 691

Clayton, D. D. 1968, Principles of Stellar Evolution and Nucleosynthesis (New York: McGraw-Hill)

Corliss, C. H., \& Bozman, W. R. 1962, Experimental Transition Probabilities for Spectral lines of Seventy Elements (NBS Monograph 53; Washington: GPO)

Cowley, C. R., \& Corliss, C. H. 1983, MNRAS, 203, 651

Danzmann, K., \& Kock, M. 1980, J. Phys. B, 13, 2051

Davidson, M. D., Snoek, L. C., Volten, H., \& Dönszelmann, A. 1992, A\&A, 255,457

Froese Fisher, C. 1975, Canadian J. Phys., 53, 184

Fuhr, J. R., Martin, G. A., \& Wiese, W. L. 1988, J. Phys. Chem. Ref. Data, 17, Suppl. 4

Fujimoto, M. Y., Ikeda, Y., \& Iben, I., Jr. 2000, ApJ, 529, L25

Gallino, R., Arlandini, C., Busso, M., Lugaro, M., Travaglio, C., Straniero, O., Chieffi, A., \& Limongi, M. 1998, ApJ, 497, 388

Gartz, T. 1973, A\&A, 26, 471

Goly, A., Kusz, J., Nguyen Quang, B., \& Weniger, S. 1991, J. Quant. Spectrosc. Radiat. Transfer, 45, 157

Gratton, R. G., Carretta, E., Eriksson, K., \& Gustafsson, B. 1999, A\&A, 350,955

Grevesse, N., Blackwell, D. E., \& Petford, A. D. 1989, A\&A, 208, 157

Gustafsson, B., Karlsson, T., Olsson, E., Edvardsson, B., \& Ryde, N. 1999, A\&A, 342, 426

Hannaford, P., Lowe, R. M., Grevesse, N., Biémont, E., \& Whaling, W. 1982, ApJ, 261, 736

Hannaford, P., Lowe, R. M., Grevesse, N., \& Noels, A. 1992, A\&A, 259, 301

Heise, C., \& Kock, M. 1990, A\&A, 230, 244

Hill, V., Barbuy, B., Spite, M., Spite, F., Cayrel, R., Plez, B., Beers, T. C., Nortström, B., \& Nissen, P. E. 2000, A\&A, 353, 557

Hollowell, D., Iben, I., Jr., \& Fujimoto, M. Y. 1990, ApJ, 351, 245

Kostyk, R. I., \& Orlova, T. V. 1983, Astrometriya Astrofiz., 49, 39

Kroll, S., \& Kock, M. 1987, A\&AS, 67, 225

Kurucz, R. L. 1993a, CD-ROM 13, ATLAS9 Stellar Atmospheres Programs and $2 \mathrm{~km} / \mathrm{s}$ Grid (Cambridge: Smithsonian Astrophys. Obs.)

. 1993b, CD-ROM 18, SYNTHE Spectrum Synthesis Programs and Line Data (Cambridge: Smithsonian Astrophys. Obs.)

Kurucz, R. L., \& Bell, B. 1995, CD-ROM 23, Atomic Line List (Cambridge: Smithsonian Astrophys. Obs.)

Kurucz, R. L., \& Peytremann, E. 1975, SAO Special Report 362 (Cambridge: Smithsonian Inst.)

Luck, R. E., \& Bond, H. E. 1981, ApJ, 244, 919 
Maier, R. S., \& Whaling, W. 1977, J. Quant. Spectrosc. Radiat. Transfer, 18,501

Martin, G. A., Fuhr, J. R., \& Wiese, W. L. 1988, J. Phys. Chem. Ref. Data, 17, Suppl. 3

McClure, R. D., \& Woodsworth A. W. 1990, ApJ, 352, 709

McWilliam, A. 1998, AJ, 115, 1640

McWilliam, A., Preston, G. W., Sneden, C., \& Searle, L. 1995, AJ, 109, 2757

Moity, J. 1983, A\&AS, 52, 37

Norris, J. E., Ryan, S. G., \& Beers, T. C. 1996, ApJS, 107, 391 .1997a, ApJ, 488, 350 1997b, ApJ, 489, L169 2001, ApJ, 561, 1034

O'Brian, T. R., Wickliffe, M. E., Lawler, J. E., Whaling, W., \& Brault, J. W. 1991, J. Opt. Soc. Am. B, 8, 1185

Peterson, R. C., \& Carney, B. W. 1989, ApJ, 347, 266

Preston, G. W., \& Sneden, C. 2001, AJ, in press

Qian, Y.-Z., \& Wasserburg, G. J. 2001, ApJ, 552, L55

Roberts, J. R., Andersen, T., \& Sorensen, G. 1973, ApJ, 181, 567

Rossi, S., Beers, T. C., \& Sneden, C. 1999, in ASP Conf. Ser. 165, Third Stromlo Meeting, ed. B. Gibson, T. Axelrod, \& M. Putman (San Francisco: ASP), 268

Ryan, S. G. 1998, A\&A, 331, 1051

Ryan, S. G., Aoki, W., Norris, J. E., Beers, T. C., Gallino, R., Busso, M., \& Ando, H. 2001, Nucl. Phys. A, 688, 209C

Ryan, S. G., Norris, J. E., \& Beers, T. C. 1996, ApJ, 471, 254
Ryan, S. G., Norris, J. E., \& Bessell, M. S. 1991, AJ, 102, 303

Smith, G., \& O’Neill, J. A. 1975, A\&A, 38, 1

Sneden, C., Cowan, J. J., Ivans, I. I., Fuller, G. M., Burles, S., Beers, T. C., \& Lawler, J. E. 2000, ApJ, 533, L139

Sneden, C., McWilliam, A., Preston, G. W., Cowan, J. J., Burris, D., \& Armosky, B. J. 1996, ApJ, 467, 819

Spite, M., \& Spite, F. 1978, A\&A, 67, 23

Thévenin, F. 1989, A\&AS, 77, 137

Tomkin, J., Lemke, M., Lambert, D. L., \& Sneden, C. 1992, AJ, 104, 1568

Tomkin, J., Woolf, V. M., Lambert, D. L., \& Lemke, M. 1995, AJ, 109, 2204

Truran, J. W. 1981, A\&A, 97, 391

Unsöld, A. 1955, Physik der Sternatmosphären (2d Ed.; Berlin: Springer)

Vanture, A. 1992, AJ, 103, 2035

Ward, L., Vogel, O., Arnesen, A., Hallin, R., \& Wännström, A. 1985, Phys. Scr., 31,161

Westin, J., Sneden, C., Gustafsson, B., \& Cowan, J. J. 2000, ApJ, 530, 783

Wiese, W. L. 1983, Highlights Astron., 6, 795

Wiese, W. L., \& Fuhr, J. R. 1975, J. Phys. Chem. Ref. Data, 4, 263

Wiese, W. L., \& Martin, G. A. 1980, Wavelengths and Transition Probabilities for Atoms and Atomic Ions (NSRDS-NBS No. 68, Part II; Washington: GPO)

Wiese, W. L., Smith, M. W., \& Miles, B. M. 1969, Atomic Transition Probabilities, Vol. 2, Sodium through Calcium (NSRDS-NBS 22; Washington: GPO) 